\title{
The Geometry of Linear Higher-Order Recursion*
}

\author{
Ugo Dal Lago \\ Dipartimento di Scienze dell'Informazione \\ Università degli Studi di Bologna, Italy \\ dallago@cs.unibo.it
}

\begin{abstract}
Linearity and ramification constraints have been widely used to weaken higher-order (primitive) recursion in such a way that the class of representable functions equals the class of polytime functions, as the works by Leivant, Hofmann and others show. This paper shows that fine-tuning these two constraints leads to different expressive strengths, some of them lying well beyond polynomial time. This is done by introducing a new semantics, called algebraic context semantics. The framework stems from Gonthier's original work and turns out to be a versatile and powerful tool for the quantitative analysis of normalization in the lambda-calculus with constants and higher-order recursion.
\end{abstract}

\section{Introduction}

Implicit computational complexity aims at giving machine-independent characterizations of complexity classes. In recent years, the field has produced a number of interesting results. Many of them relate complexity classes to function algebras, typed lambda calculi and logics by introducing appropriate restrictions to (higher-order) primitive recursion or second-order linear logic. The resulting subsystems are then shown to correspond to complexity classes by way of a number of different, heterogeneous techniques. Many kinds of constraints have been shown to be useful in this context; this includes ramification [3, 20, 22], linear types [17, 4, 21, 8] and restricted exponentials [14, 19]. However, the situation is far from being satisfactory. There are still many open problems: for example, it is not yet clear what the consequences of combining different constraints are. Moreover, using such systems as a foundation for resource-aware programming languages relies heavily on them to be able to capture interesting algorithms. Despite some recent progresses [16 5], a lot of work still has to be done. Undoubtedly, what is still lacking in this field is a powerful and simple mathematical framework for the analysis of quantitative aspects of computation. Indeed, existing systems have been often studied using ad-hoc techinques which cannot be easily adapted to other systems. A unifying framework would not just make the task of proving correspondences between systems and complexity classes simpler, but could be possibly used itself as a basis for introducing resource-consciousness into programming languages. We believe that ideal candidates to pursue these goals are Girard's geometry of interaction [13, 12] and related frameworks, such as context semantics [15, 26]. Using the above techniques as tools in the study of complexity of normalization has already been done by Baillot and Pedicini in the context of elementary linear logic [2], while game models being fully abstract with respect to operational theory of improvement [28] have recently been proposed by Ghica [11]. Ordinal analysis has already been proved useful to the study of ramified systems (e.g. [27 [29]) but, to the author's knowledge, the underlying framework has not been applied to linear calculi. Similarly, Leivant's instrinsic reasoning framework [23 24] can help defining and studying restrictions on first-order arithmetic inducing complexity bounds on provably total functions: however, the consequences of linearity conditions cannot be easily captured and studied in the framework.

In this paper, we introduce a new semantical framework for higher-order recursion, called algebraic context semantics. It is inspired by context semantics, but designed to be a tool for proving quantitative rather than qualitative properties of programs. As we will see, it turns out to be of great help when analyzing quantitative aspects of normalization in presence of linearity and ramification constraints. Informally, algebraic context semantics allows to prove bounds on the algebraic potential size

\footnotetext{
*The author is partially supported by PRIN projects PROTOCOLLO (2002) and FOLLIA (2004).
} 
of System T terms, where the algebraic potential size of any term $M$ is the maximum size of free algebra terms which appear as subterms of reducts of $M$. As a preliminary result, the algebraic potential size is shown to be a bound to normalization time, modulo a polynomial overhead. Consequently, bounds obtained through context semantics translate into bounds to normalization time.

Main results of this work are sharp characterizations of the expressive power of various fragments of System T. Almost all of them are novel. Noticeably, these results are obtained in a uniform way and, as a consequence, most of the involved work has been factorized over the subsystems and done just once. Moreover, we do not simply prove that the class of representable first-order functions equals complexity classes but, instead, we give bounds on the time needed to normalize any term. This makes our results stronger than similar ones from the literature [17, 4, 21]. Our work gives some answers to a fundamental question implicitly raised by Hofmann [17]: are linearity conditions sufficient to keep the expressive power of higher-order recursion equal to that of first-order recursion? In particular, a positive answer can be given in case ramification does not hold. The methodology introduced here can be applied to multiplicative and exponential linear logic [6], allowing to reprove soundness results for various subsystems of the logic.

The rest of the paper is organized as follows: in Section 2 a call-by-value lambda calculus will be described as well as an operational semantics for it; in Section 3 we will define ramification and linearity conditions on the underlying type system, together with subsystems induced by these constraints; in Section 4 we motivate and introduce algebraic context semantics, while in Section 5 we will use it to give bounds on the complexity of normalization. Section 6 is devoted to completeness results.

\section{Syntax}

In this section, we will give some details on our reference system, namely a formulation of Gödel's T in the style of Matthes and Joachimsky [18]. The definitions will will be standard. The only unusual aspect of our syntax is the adoption of weak call-by-value reduction. This will help in keeping the language of terms and the underlying type system simpler.

Data will be represented by terms in some free algebras. As it will be shown, different free algebras do not necessarily behave in the same way from a complexity viewpoint, as opposed to what happens in computability theory. As a consequence, we cannot restrict ourselves to a canonical free algebra and need to keep all of them in our framework. A free algebra $\mathbb{A}$ is a couple $\left(\mathcal{C}_{\mathbb{A}}, \mathcal{R}_{\mathbb{A}}\right)$ where $\mathcal{C}_{\mathbb{A}}=\left\{c_{1}^{\mathbb{A}}, \ldots, c_{k(\mathbb{A})}^{\mathbb{A}}\right\}$ is a finite set of constructors and $\mathcal{R}_{\mathbb{A}}: \mathcal{C}_{\mathbb{A}} \rightarrow \mathbb{N}$ maps every constructor to its arity. If the underlying free algebra $\mathbb{A}$ is clear from the context, we simply write $\mathcal{R}(c)$ in place of $\mathcal{R}_{\mathbb{A}}(c)$. A free algebra $\mathbb{A}=\left(\left\{c_{1}^{\mathbb{A}}, \ldots, c_{k(\mathbb{A})}^{\mathbb{A}}\right\}, \mathcal{R}_{\mathbb{A}}\right)$ is a word algebra if

- $\mathcal{R}\left(c_{i}^{\mathbb{A}}\right)=0$ for one (and only one) $i \in\{1, \ldots, k(\mathbb{A})\}$;

- $\mathcal{R}\left(c_{j}^{\mathbb{A}}\right)=1$ for every $j \neq i$ in $\{1, \ldots, k(\mathbb{A})\}$.

If $\mathbb{A}=\left(\left\{c_{1}^{\mathbb{A}}, \ldots, c_{k(\mathbb{A})}^{\mathbb{A}}\right\}, \mathcal{R}_{\mathbb{A}}\right)$ is a word algebra, we will assume $c_{k(\mathbb{A})}^{\mathbb{A}}$ to be the distinguished element of $\mathcal{C}_{\mathbb{A}}$ whose arity is 0 and $c_{1}^{\mathbb{A}}, \ldots, c_{k(\mathbb{A})-1}^{\mathbb{A}}$ will denote the elements of $\mathcal{C}_{\mathbb{A}}$ whose arity is 1 . $\mathbb{U}=\left(\left\{c_{1}^{\mathbb{U}}, c_{2}^{\mathbb{U}}\right\}, \mathcal{R}_{\mathbb{U}}\right)$ is the word algebra of unary strings. $\mathbb{B}=\left(\left\{c_{1}^{\mathbb{B}}, c_{2}^{\mathbb{B}}, c_{3}^{\mathbb{B}}\right\}, \mathcal{R}_{\mathbb{B}}\right)$ is the word algebra of binary strings. $\mathbb{C}=\left(\left\{c_{1}^{\mathbb{C}}, c_{2}^{\mathbb{C}}\right\}, \mathcal{R}_{\mathbb{C}}\right)$, where $\mathcal{R}_{\mathbb{C}}\left(c_{1}^{\mathbb{C}}\right)=2$ and $\mathcal{R}_{\mathbb{C}}\left(c_{2}^{\mathbb{C}}\right)=0$ is the free algebra of binary trees. $\mathbb{D}=\left(\left\{c_{1}^{\mathbb{D}}, c_{2}^{\mathbb{D}}, c_{3}^{\mathbb{D}}\right\}, \mathcal{R}_{\mathbb{D}}\right)$, where $\mathcal{R}_{\mathbb{D}}\left(c_{1}^{\mathbb{D}}\right)=\mathcal{R}_{\mathbb{D}}\left(c_{2}^{\mathbb{D}}\right)=2$ and $\mathcal{R}_{\mathbb{D}}\left(c_{3}^{\mathbb{D}}\right)=0$ is the free algebra of binary trees with binary labels. Natural numbers can be encoded by terms in $\mathbb{U}:\ulcorner 0\urcorner=c_{2}^{\mathbb{U}}$ and $\ulcorner n+1\urcorner=c_{1}^{\mathbb{U}}\ulcorner n\urcorner$ for all $n$. In the same vein, elements of $\{0,1\}^{*}$ are in one-to-one correspondence to terms in $\mathbb{B}:\ulcorner\varepsilon\urcorner=c_{3}^{\mathbb{B}}$, and for all $s \in\{0,1\}^{*}$, $\ulcorner 0 s\urcorner=c_{1}^{\mathbb{B}}\ulcorner s\urcorner$ and $\ulcorner 1 s\urcorner=c_{2}^{\mathbb{B}}\ulcorner s\urcorner$. When this does not cause ambiguity, $\mathcal{C}_{\mathbb{A}}$ and $\mathcal{R}_{\mathbb{A}}$ will be denoted by $\mathcal{C}$ and $\mathcal{R}$, respectively. $\mathscr{A}$ will be a fixed, finite family $\left\{\mathbb{A}_{1}, \ldots, \mathbb{A}_{n}\right\}$ of free algebras whose constructor sets $\mathcal{C}_{\mathbb{A}_{1}}, \ldots, \mathcal{C}_{\mathbb{A}_{n}}$ are assumed to be pairwise disjoint. We will hereby assume $\mathbb{U}, \mathbb{B}, \mathbb{C}$ and $\mathbb{D}$ to be in $\mathscr{A} . \mathscr{K}_{\mathscr{A}}$ is the maximum arity of constructors of free algebras in $\mathscr{A}$, i.e. the natural number

$$
\max _{\mathbb{A} \in \mathscr{A}} \max _{c \in \mathcal{C}_{\mathbb{A}}} \mathcal{R}_{\mathbb{A}}(c) .
$$

$\mathscr{E}_{\mathbb{A}}$ is the set of terms for the algebra $\mathbb{A}$, while $\mathscr{E}_{\mathscr{A}}$ is the union of $\mathscr{E}_{\mathbb{A}}$ over all algebras $\mathbb{A}$ in $\mathscr{A}$.

Programs will be written in a fairly standard lambda calculus with constants (corresponding to free algebra constructors) and recursion. The latter will not be a combinator but a term former, as in [18]. Moreover, we will use a term former for conditional, keeping it distinct from the one for recursion. This apparent redundancy is actually needed in presence of ramification (see, for example, [20]). The language $\mathscr{M}_{\mathscr{A}}$ of terms is defined by the following productions:

$$
M::=x|c| M M|\lambda x . M| M\{\{M, \ldots, M\}\} \mid M\langle\langle M, \ldots, M\rangle\rangle
$$




$$
\begin{aligned}
& \overline{x: A \vdash x: A} A \quad \frac{\Gamma \vdash M: B}{\Gamma, x: A \vdash M: B} W \quad \frac{\Gamma, x: A, y: A \vdash M: B}{\Gamma, z: A \vdash M\{z / x, z / y\}: B} C
\end{aligned}
$$

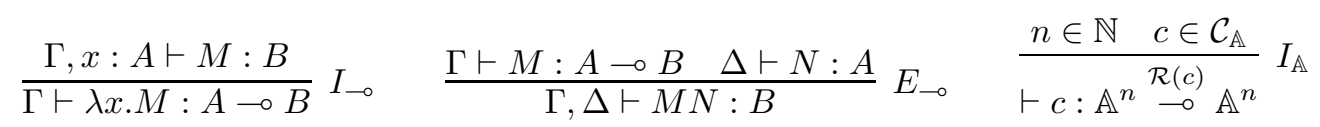

$$
\begin{aligned}
& \frac{\Gamma_{i} \vdash M_{c_{i}^{\mathbb{A}}}: \mathbb{A}^{m} \stackrel{\mathcal{R}\left(c_{i}^{\mathrm{A}}\right)}{\longrightarrow} C \Delta \vdash L: \mathbb{A}^{m}}{\Gamma_{1}, \ldots, \Gamma_{n}, \Delta \vdash L\left\{\left\{M_{c_{1}^{\mathbb{A}}} \cdots M_{\left.c_{k(\mathbb{A})}^{\mathrm{A}}\right)}\right\}\right\}: C} E_{\mathbb{A}}^{C} \quad \frac{\Gamma_{i} \vdash M_{c_{i}^{\mathbb{A}}}: \mathbb{A}^{m} \stackrel{\mathcal{R}\left(c_{i}^{\mathbb{A}}\right)}{\longrightarrow} C \stackrel{\mathcal{R}\left(c_{i}^{\mathbb{A}}\right)}{\longrightarrow} C \quad \Delta \vdash L: \mathbb{A}^{m}}{\Gamma_{1}, \ldots, \Gamma_{n}, \Delta \vdash L\left\langle\left\langle M_{c_{1}^{\mathbb{A}}} \cdots M_{\left.c_{k(\mathbb{A})}^{\mathrm{A}}\right)}\right\rangle: C\right.} E_{\mathbb{A}}^{R}
\end{aligned}
$$

Figure 1. Type assignment rules

where $c$ ranges over the constructors for the free algebras in $\mathscr{A}$. Term formers $\cdot\{\{\cdot, \ldots, \cdot\}\}$ and $\cdot\langle\langle\cdot, \ldots, \cdot\rangle\rangle$ are conditional and recursion term formers, respectively.

The language $\mathscr{T}_{\mathscr{A}}$ of types is defined by the following productions:

$$
A::=\mathbb{A}^{n} \mid A \multimap A
$$

where $n$ ranges over $\mathbb{N}$ and $\mathbb{A}$ ranges over $\mathscr{A}$. Indexing base types is needed to define ramification conditions as in [20]; $\mathbb{A}^{n}$, in particular, is not a cartesian product. The notation $A \stackrel{n}{\circ} B$ is defined by induction on $n$ as follows: $A \stackrel{0}{\longrightarrow} B$ is just $B$, while $A \stackrel{n+1}{\rightarrow} B$ is $A \multimap(A \stackrel{n}{\circ} B)$. The level $V(A) \in \mathbb{N}$ of a type $A$ is defined by induction on $A$ :

$$
\begin{aligned}
V\left(\mathbb{A}^{n}\right) & =n ; \\
V(A \multimap B) & =\max \{V(A), V(B)\} .
\end{aligned}
$$

When this does not cause ambiguity, we will denote a base type $\mathbb{A}^{n}$ simply by $\mathbb{A}$.

The rules in Figure 1define the assignment of types in $\mathscr{T}_{\mathscr{A}}$ to terms in $\mathscr{M}_{\mathscr{A}}$. A type derivation $\pi$ with conclusion $\Gamma \vdash M: A$ will be denoted by $\pi: \Gamma \vdash M: A$. If there is $\pi: \Gamma \vdash M: A$ then we will mark $M$ as a typeable term. A type derivation $\pi: \Gamma \vdash M: A$ is in standard form if the typing rule $W$ is used only when necessary, i.e. immediately before an instance of $I_{-}$. We will hereby assume to work with type derivations in standard form. This restriction does not affect the class of typeable terms.

The recursion depth $R(\pi)$ of a type derivation $\pi: \Gamma \vdash M: A$ is the biggest number of $E_{\mathbb{A}}^{R}$ instances on any path from the root to a leaf in $\pi$. The highest tier $I(\pi)$ of a type derivation $\pi: \Gamma \vdash M: A$ is the maximum integer $i$ such that there is an instance

$$
\frac{\pi_{1} \quad \ldots \quad \pi_{n} \quad \Delta \vdash L: \mathbb{A}^{i}}{\Gamma, \Delta \vdash L\left\langle\left\langle M_{1}, \ldots, M_{n}\right\rangle\right\rangle: C} E_{\mathbb{A}}^{R}
$$

of $E_{\AA}^{R}$ inside $\pi$.

Values are defined by the following productions:

$$
\begin{aligned}
& V::=x|\lambda x . M| T ; \\
& T::=c \mid T T .
\end{aligned}
$$

where $c$ ranges constructors. Reduction is weak and call-by-value. The reduction rule $\rightarrow$ on $\mathscr{M}_{\mathscr{A}}$ is given in Figure 2 We will forbid firing a redex under an abstraction or inside a recursion or a conditional. In other words, we will define $\sim$ from $\rightarrow$ by the following set of rules:

$$
\begin{aligned}
& \frac{M \rightarrow N}{M \leadsto N} \quad \frac{M \leadsto N}{M L \leadsto N L} \quad \frac{M \leadsto N}{L M \leadsto L N} \\
& \frac{M \leadsto N}{M\left\{\left\{L_{1}, \ldots, L_{n}\right\}\right\} \leadsto N\left\{\left\{L_{1}, \ldots, L_{n}\right\}\right\}} \quad \frac{M \leadsto N}{M\left\langle\left\langle L_{1}, \ldots, L_{n}\right\rangle\right\rangle \leadsto N\left\langle\left\langle L_{1}, \ldots, L_{n}\right\rangle\right\rangle}
\end{aligned}
$$




$$
\begin{aligned}
(\lambda x . M) V & \rightarrow M\{V / x\} \\
c_{i}^{\mathbb{A}}\left(t_{1}, \ldots, t_{\mathcal{R}\left(c_{i}^{\mathrm{A}}\right)}\right)\left\{\left\{M_{c_{1}^{\mathrm{A}}}, \ldots, M_{c_{k}^{\mathrm{A}}}\right\}\right\} & \rightarrow M_{c_{i}^{\mathrm{A}}} t_{1} \cdots t_{\mathcal{R}\left(c_{i}\right)} \\
c_{i}^{\mathbb{A}}\left(t_{1}, \ldots, t_{\mathcal{R}\left(c_{i}^{\mathrm{A}}\right)}\right)\left\langle\left\langle M_{c_{1}^{\mathrm{A}}}, \ldots, M_{c_{k}^{\mathrm{A}}}\right\rangle\right\rangle & \rightarrow M_{c_{i}^{\mathrm{A}}} t_{1} \cdots t_{\mathcal{R}\left(c_{i}^{\mathrm{A}}\right)}\left(t_{1}\left\langle\left\langle M_{c_{1}^{\mathrm{A}}}, \ldots, M_{c_{k}^{\mathrm{A}}}\right\rangle\right) \cdots\left(t_{\mathcal{R}\left(c_{i}^{\mathrm{A}}\right)}\left\langle\left\langle M_{c_{1}^{\mathrm{A}}}, \ldots, M_{c_{k}^{\mathrm{A}}}\right\rangle\right\rangle\right)\right.
\end{aligned}
$$

Figure 2. Normalization on terms

Redexes in the form $(\lambda x . M) V$ are called beta redexes; those like $t\left\{\left\{M_{1}, \ldots, M_{n}\right\}\right\}$ are called conditional redexes; those in the form $t\left\langle\left\langle M_{1}, \ldots, M_{n}\right\rangle\right\rangle$ are recursive redexes. The argument of the beta redex $(\lambda x . M) V$ is $V$, while that of $t\left\{\left\{M_{1}, \ldots, M_{n}\right\}\right\}$ and $t\left\langle\left\langle M_{1}, \ldots, M_{n}\right\rangle\right\rangle$ is $t$. As usual, $\sim^{*}$ and $\sim^{+}$denote the reflexive and transitive closure of $\leadsto$ and the transitive closrue of $\leadsto$, respectively.

Proposition 1 If $\vdash M: \mathbb{A}^{n}$, then the (unique) normal form of $M$ is a free algebra term $t$.

Proof. In this proof, terms from the grammar $T::=c \mid T T$ (where $c$ ranges over constructors) are dubbed algebraic. We prove the following stronger claim by induction on $M:$ if $\vdash M: A$ and $M$ is a normal form, then it must be a value. We distinguish some cases:

- A variable cannot be typed in the empty context, so $M$ cannot be a variable.

- If $M$ is a constant or an abstraction, then it is a value by definition.

- If $M$ is an application $N L$, then there is a type $B$ such that both $\vdash N: B \multimap A$ and $\vdash L: B$. By induction hypothesis both $N$ and $L$ must be values. But $N$ cannot be an abstraction (because otherwise $N L$ would be a redex) nor a variable (because a variable cannot be typed in the empty context). As a consequence, $N$ must be algebraic. Every algebraic term, however, has type $\mathbb{A}^{i} \stackrel{n}{\circ} \mathbb{A}^{i}$ where $n \geq 0$. Clearly, this implies $n \geq 1$ and $B=\mathbb{A}^{i}$. This, in turn, implies $L$ to be algebraic (it cannot be a variable nor an abstraction). So, $M$ is itself algebraic.

- If $M$ is $N\left\{\left\{M_{1}, \ldots, M_{n}\right\}\right\}$, then $N$ must be a value such that $\vdash N: \mathbb{A}^{i}$. As a consequence, it must be a free algebraic term $t$. But this is a contraddiction, since $M$ is assumed to be a value.

- If $M$ is $N\left\langle\left\langle M_{1}, \ldots, M_{n}\right\rangle\right\rangle$, then we can proceed exactly as in the previous case.

This concludes the proof, since the relation $\sim$ enjoys a one-step diamond property (see [7]).

It should be now clear that the usual recursion combinator $\mathbf{R}$ can be retrieved by putting $\mathbf{R}=\lambda x . \lambda y_{1} \ldots . . \lambda y_{n} . x\left\langle\left\langle y_{1}, \ldots, y_{n}\right\rangle\right\rangle$. The size $|M|$ of a term $M$ is defined as follows by induction on the structure of $M$ :

$$
\begin{aligned}
|x|=|c| & =1 \\
|\lambda x . M| & =|M|+1 \\
|M N| & =|M|+|N| \\
\left|M\left\langle\left\langle M_{1}, \ldots, M_{n}\right\rangle\right\rangle\right|=\left|M\left\{\left\{M_{1}, \ldots, M_{n}\right\}\right\}\right| & =|M|+\left|M_{1}\right|+\ldots+\left|M_{n}\right|+n
\end{aligned}
$$

Notice that, in particular, $|t|$ equals the number of constructors in $t$ for every free algebra term $t$.

\section{Subsystems}

The system, as it has been just defined, is equivalent to Gödel System T and, as a consequence, its expressive power equals the one of first-order arithmetic. We are here interested in two different conditions on programs, which can both be expressed as constraints on the underlying type-system:

- First of all, we can selectively enforce linearity by limiting the applicability of contraction rule $C$ to types in a class $\mathrm{D} \subseteq \mathscr{T}_{\mathscr{A}}$. Accordingly, the constraint $\operatorname{cod}\left(\Gamma_{i}\right) \subseteq \mathrm{D}$ must be satisfied in rule $E_{\mathbb{A}}^{R}$ (for every $i \in\{1, \ldots, n\}$ ). In this way, we obtain a system $\mathrm{H}(\mathrm{D})$. As an example, $\mathrm{H}(\emptyset)$ is a system where rule $C$ is not allowed on any type and contexts $\Gamma_{i}$ are always empty in rule $E_{\mathbb{A}}^{R}$.

- Secondly, we can introduce a ramification condition on the system. This can be done in a straightforward way by adding the premise $m>V(C)$ to rule $E_{\mathbb{A}}^{R}$. This corresponds to impose the tier of the recurrence argument to be strictly higher 
than the tier of the result (analogously to Leivant [20]). Indeed, $m$ is the integer indexing the type of the recurrence argument, while $V(C)$ is the maximum integer appearing as an index in $C$, which is the type of the result. For every system $H(D)$, we obtain in this way a ramified system $R H(D)$.

The constraint $\operatorname{cod}\left(\Gamma_{i}\right) \subseteq \mathrm{D}$ in instances of rule $E_{\mathbb{A}}^{R}$ is needed to preserve linearity during reduction: if $c_{i}^{\mathbb{A}} t\left\langle\left\langle M_{1}, \ldots, M_{k(\mathbb{A})}\right\rangle\right\rangle$ is a recursive redex where $M_{i}$ has a free variable $x$ of type $A \notin \mathrm{D}$, firing the redex would produce a term with two occurrences of $x$.

Let us define the following two classes of types:

$$
\begin{aligned}
\mathrm{W} & =\left\{\mathbb{A}^{n} \mid \mathbb{A} \in \mathscr{A} \text { is a word algebra }\right\} \\
\mathrm{A} & =\left\{\mathbb{A}^{n} \mid \mathbb{A} \in \mathscr{A}\right\} .
\end{aligned}
$$

In the rest of this paper, we will investigate the expressive power of some subsytems $H(D)$ and $R H(D)$ where $D \subseteq A$. The following table reports the obtained results:

\begin{tabular}{|c|c|c|c|}
\cline { 2 - 4 } \multicolumn{1}{c|}{} & A & W & $\emptyset$ \\
\hline \hline $\mathrm{H}(\cdot)$ & FR & FR & FR \\
\hline $\mathrm{RH}(\cdot)$ & FE & FP & FP \\
\hline \hline
\end{tabular}

Here, FP (respectively, FE) is the class of functions which can be computed in polynomial (respectively, elementary) time. FR, on the other hand, is the class of (first-order) primitive recursive functions, which equals the class of functions which can be computed in time bounded by a primitive recursive function. For example, $\mathrm{RH}(\mathrm{A})$ is proved sound and complete with respect to elementary time, while $\mathrm{H}(\emptyset)$ is shown to capture (first-order) primitive recursion.

Forbidding contraction on higher-order types is quite common and has been extensively used as a tool to restrict the class of representable functions inside System T [17, 4, 21]. The correspondence between $\mathrm{RH}(\mathrm{W})$ and $\mathbf{F P}$ is well known from the literature [17 4], although in a slightly different form. To the author's knowledge, all the other charaterization results are novel. Similar results can be ascribed to Leivant and Marion [25] 22], but they do not take linearity constraints into account. Notice that, in presence of ramification, going from $W$ to $A$ dramatically increases the expressive power, while going from $W$ to $\emptyset$ does not cause any loss of expressivity. The "phase-transition" occurring when switching from $R H(W)$ to $R H(A)$ is really surprising, since the only difference between these two systems are the class of types to which linearity applies: in one case we only have word algebras, while in the other case we have all free algebras.

\section{Algebraic Context Semantics}

In this section, we will introduce algebraic context semantics, showing how bounds on the normalization time of a any term $M$ can be inferred from its semantics.

The first result we need relates the complexity of normalizing any given term $M$ to the size of free algebra terms appearing as subterms of reducts of $M$. The algebraic potential size $A(M)$ of a typable term $M$ is the maximum natural number $n$ such that $M \sim^{*} N$ and there is a redex in $N$ whose argument is a free algebra term $t$ with $|t|=n$. Since the calculus is strongly normalizing, there is always a finite bound to the size of reducts of a term and, as a consequence, the above definition is well-posed. According to the following result, the algebraic potential size of a term $M$ such that $\pi: \Gamma \vdash_{\mathrm{H}(\mathrm{A})} M: A$ is an overestimate on the time needed to normalize the term (modulo some polynomials that only depends on $R(\pi)$ ):

Proposition 2 For every $d \in \mathbb{N}$ there are polynomials $p_{d}, q_{d}: \mathbb{N}^{2} \rightarrow \mathbb{N}$ such that whenever $\pi: \Gamma \vdash_{\mathrm{H}(\mathrm{A})} M: A$ and $M \sim^{n} N$, then $n \leq p_{R(\pi)}(|M|, A(M))$ and $|N| \leq q_{R(\pi)}(|M|, A(M))$.

Proof. Let us first observe that the number of recursive redexes fired during normalization of $M$ is bounded by $s_{R(\pi)}(|M|, A(M))$, where

$$
s_{d}(x, y)=x y^{d}
$$

Indeed, consider subterms of $M$ in the form $L\left\langle\left\langle N_{1}, \ldots, N_{k}\right\rangle\right\rangle$. Clearly, there are at most $|M|$ such terms. Moreover, each such subterm can result in at most $|A(M)|^{R(\pi)}$ recursive redexes. Indeed, it can be copied at most $|A(M)|^{R(\pi)-1}$ times, and each copy can itself result in $|A(M)|$ recursive redexes. Now, notice that firing a beta or a conditional redex does not increase the number of variable occurrences in the term. Conversely, firing a recursive redex can make it bigger by at most $|M|$. We can conclude that the number of beta redexes in the form $(\lambda x . M) t$ (let us call them algebraic redexes) is at most 
$|M| s_{R(\pi)}(|M|, A(M))$ and, moreover, they can make the term to increase in size by at most $A(M)|M| s_{R(\pi)}(|M|, A(M))$ altogether. Firing a recursive redex

$$
c_{i}\left(t_{1}, \ldots, t_{\mathcal{R}\left(c_{i}\right)}\right)\left\langle\left\langle M_{c_{1}}, \ldots, M_{c_{k}}\right\rangle\right\rangle
$$

can make the size of the underlying term to increase by $r_{R(\pi)}(|M|, A(M))$ where

$$
r_{R(\pi)}(x, y)=\mathscr{K}_{\mathscr{A}}(y+x+x y) .
$$

Indeed:

$$
\begin{aligned}
& \mid M_{c_{i}} t_{1} \cdots t_{\mathcal{R}\left(c_{i}\right)}\left(t_{1}\left\langle\left\langle M_{c_{1}}, \ldots, M_{c_{k}}\right\rangle\right\rangle\right) \cdots\left(t_{\mathcal{R}\left(c_{i}\right)}\left\langle\left\langle M_{c_{1}}, \ldots, M_{c_{k}}\right\rangle\right) \mid\right. \\
= & \left|M_{c_{i}} t_{1} \cdots t_{\mathcal{R}\left(c_{i}\right)}\right|+\left|t_{1}\left\langle\left\langle M_{c_{1}}, \ldots, M_{c_{k}}\right\rangle\right)\right|+\ldots+\left|t_{\mathcal{R}\left(c_{i}\right)}\left\langle\left\langle M_{c_{1}}, \ldots, M_{c_{k}}\right\rangle\right\rangle\right| \\
\leq & \mid c_{i}\left(t_{1}, \ldots, t_{\mathcal{R}\left(c_{i}\right)}\right)\left\langle\left\langle M_{c_{1}}, \ldots, M_{c_{k}}\right\rangle\right|+\sum_{i=1}^{\mathcal{R}\left(c_{i}\right)}\left(\left|t_{i}\right|+\left|M_{c_{1}}\right|+\ldots+\left|M_{c_{k}}\right|+k\right) \\
\leq & \mid c_{i}\left(t_{1}, \ldots, t_{\mathcal{R}\left(c_{i}\right)}\right)\left\langle\left\langle M_{c_{1}}, \ldots, M_{c_{k}}\right\rangle\right|+\mathscr{K}_{\mathscr{A}}(A(M)+|M|+A(M)|M|)
\end{aligned}
$$

because $\left|M_{c_{1}}\right|+\ldots+\left|M_{c_{k}}\right|+k$ is bounded by $|M|+A(M)|M|$ and $\left|c_{i}\left(t_{1}, \ldots, t_{\mathcal{R}\left(c_{i}\right)}\right)\right|$ is bounded by $A(M)$. We can now observe that firing any redex other than algebraic or recursive ones makes the size of the term to strictly decrease. As a consequence, we can argue that

$$
\begin{aligned}
& q_{d}(x, y)=x+s_{d}(x, y) x y+s_{d}(x, y) r_{d}(x, y) \\
& p_{d}(x, y)=s_{d}(x, y) x+s_{d}(x, y)+q_{d}(x, y) .
\end{aligned}
$$

This concludes the proof.

Observe that in the statement of Proposition 2 it is crucial to require $M$ to be typable in $\mathrm{H}(\mathrm{A})$. Indeed, it is quite easy to build simply-typed (pure) lambda terms which have exponentially big normal forms, although having null algebraic potential size.

In the rest of this section, we will develop a semantics, derived from context semantics [15] and dubbed algebraic context semantics. We will then use it to give bounds to the algebraic potential size of terms in subsystems we are interested in and use Proposition 2 to derive time bounds.

Consider the term

$$
\text { UnAdd } \equiv \lambda x \cdot \lambda y \cdot x\left\langle\left\langle\lambda w \cdot \lambda z \cdot c_{1}^{\mathbb{U}} z, y\right\rangle\right\rangle .
$$

Clearly, UnAdd $\ulcorner n\urcorner\ulcorner m\urcorner \sim^{*}\ulcorner n+m\urcorner$. UnAdd $\ulcorner 1\urcorner\ulcorner 1\urcorner$ will be used as a reference example throughout this section. A type derivation $\sigma$ for $\mathbf{U n A d d}\ulcorner 1\urcorner\ulcorner 1\urcorner$ is the following one:

$$
\begin{aligned}
& \overline{ } \overline{\vdash c_{1}^{\mathbb{U}}: \mathbb{U}^{0} \multimap \mathbb{U}^{0}} \overline{z: \mathbb{U}^{0} \vdash z: \mathbb{U}^{0}} \\
& z: \mathbb{U}^{0} \vdash c_{1}^{\mathbb{U}} z: \mathbb{U}^{0} \\
& \overline{w: \mathbb{U}^{1}, z: \mathbb{U}^{0} \vdash c_{1}^{\mathbb{U}} z: \mathbb{U}^{0}} \\
& \overline{w: \mathbb{U}^{1} \vdash \lambda z \cdot c_{1}^{\mathbb{U}} z: \mathbb{U}^{0} \multimap \mathbb{U}^{0}} \\
& \overline{\vdash \lambda w \cdot \lambda z \cdot c_{1}^{\mathbb{U}} z: \mathbb{U}^{1} \multimap \mathbb{U}^{0} \multimap \mathbb{U}^{0}} \quad \overline{y: \mathbb{U}^{0} \vdash y: \mathbb{U}^{0}} \quad \overline{x: \mathbb{U}^{1} \vdash x: \mathbb{U}^{1}} \\
& x: \mathbb{U}^{1}, y: \mathbb{U}^{0} \vdash x\left\langle\left\langle\lambda w \cdot \lambda z \cdot c_{1}^{\mathbb{U}} z, y\right\rangle\right\rangle: \mathbb{U}^{0} \\
& \overline{x: \mathbb{U}^{1} \vdash \lambda y \cdot x\left\langle\left\langle\lambda w \cdot \lambda z \cdot c_{1}^{\mathbb{U}} z, y\right\rangle\right\rangle: \mathbb{U}^{0} \multimap \mathbb{U}^{0}} \\
& \frac{\text { UnAdd }: \mathbb{U}^{1} \multimap \mathbb{U}^{0} \multimap \mathbb{U}^{0}}{\frac{\vdash \text { UnAdd }\ulcorner 1\urcorner: \mathbb{U}^{0} \multimap \mathbb{U}^{0}}{\vdash \text { UnAdd }\ulcorner 1\urcorner\ulcorner 1\urcorner: \mathbb{U}^{0}} \eta_{0} \vdash\ulcorner 1\urcorner: \mathbb{U}^{0}}
\end{aligned}
$$

where $\eta_{0}$ and $\eta_{1}$ are defined in the obvious way.

We will study the context semantics of interaction graphs, which are graphs corresponding to type derivations. Notice that we will not use interaction graphs as a virtual machine computing normal forms - they are merely a tool facilitating the study of language dynamics. More precisely, we will put every type derivation $\pi$ in correspondence to an interaction graph 


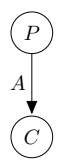

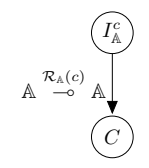

(a)

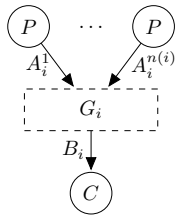

(b)

Figure 3. Base cases.

$G_{\pi}$. The context semantics of $G_{\pi}$ will be a set of trees $T\left(G_{\pi}\right)$ such that every tree $T$ in $T\left(G_{\pi}\right)$ can be associated to a term $t=L(T) \in \mathscr{E}_{\mathscr{A}}$. If $\pi: \Gamma \vdash M: A$, then $T\left(G_{\pi}\right)$ keeps track of the normalization of $M$ in the following sense: for every $t$ appearing as an argument of a reduct of $M$, there is a tree $T \in T\left(G_{\pi}\right)$ such that $t=L(T)$. Proving this property, called completeness, is the aim of Section 4.1 Completeness, together with Proposition 2 is exploited in Section 5 where bounds on normalization time for classes of terms are inferred.

Let $\mathscr{L}_{\mathscr{A}}$ be the set

$$
\left\{W, X, I_{-\circ}, E_{-}, P, C\right\} \cup \bigcup_{\mathbb{A} \in \mathscr{A}}\left\{C_{\mathbb{A}}^{N}, P_{\mathbb{A}}^{R}, C_{\mathbb{A}}^{R}\right\} \cup \bigcup_{\mathbb{A} \in \mathscr{A}} \bigcup_{c \in \mathcal{C}_{\mathbb{A}}}\left\{I_{\AA}^{c}\right\} .
$$

An interaction graph is a graph-like structure $G$. It can be defined inductively as follows: an interaction graph is either the graph in Figure 3 a) or one of those in Figure 4 where $G_{0}, G_{1}, \ldots, G_{k(\mathbb{A})}$ are themselves proof-nets as in Figure 3 b). If $G$ is an interaction graph, then $V_{G}$ denotes the set of vertices of $G, E_{G}$ denotes the set of directed edges of $G, \alpha_{G}$ is a labelling function mapping every vertex in $V_{G}$ to an element of $\mathscr{L}_{\mathscr{A}}$ and $\beta_{G}$ maps every edge in $E_{G}$ to a type in $\mathscr{T}_{\mathscr{A}}$. $\mathscr{G}_{\mathscr{A}}$ is the set of all interaction graphs.

Notice that each of the rules in figures 3 a) and 4 closely corresponds to a typing rule. Given a type derivation $\pi$, we can then build an interaction graph $G_{\pi}$ corresponding to $\pi$. For example, Figure 5 reports an interaction graph $G_{\sigma}$ where $\sigma: \vdash \mathbf{U n A d d}\ulcorner 1\urcorner\ulcorner 1\urcorner: \mathbb{U}^{0}$. Let us observe that if $\pi: \Gamma \vdash M: A$ is in standard form, then the size $\left|G_{\pi}\right|$ of $G_{\pi}$ is proportional to $|M|$.

Nodes labelled with $C$ (respectively, $P$ ) mark the conclusion (respectively, the premises) of the interaction graph. Notice that the rule corresponding to recursion (see Figure 4) allows seeing interaction graphs as nested structures, where nodes labelled with $C_{\mathbb{A}}^{R}$ and $P_{\mathbb{A}}^{R}$ delimit a box, similarly to what happens in linear logic proof-nets. If $e \in E_{G}$, then the box-premise of $e$, denoted $\theta_{G}(e)$, is the vertex labelled with $C_{\mathbb{A}}^{R}$ delimiting the box in which $e$ is contained (if such a box exists, otherwise $\theta_{G}(e)$ is undefined). If $v \in V_{G}$, the box-premise of $v$, denoted $\theta_{G}(v)$ is defined similarly. In our example (see Figure 5), $\theta_{G}\left(e_{i}\right)$ equals $v$ for every $i \leq 7$ and is otherwise undefined. If $v$ is a vertex with $\alpha_{G}(v)=C_{\mathbb{A}}^{R}$, then the recursive premise of $v$, denoted $\rho_{G}(v)$, is the edge incident to $v$ and coming from outside the box. In our example, $\rho_{G}(v)$ is $e_{12}$.

Defining algebraic context semantics requires a number of auxiliary concepts, like the one of a term context and the one of a type context. The set $\mathscr{N}_{\mathbb{A}}$ of term contexts for $\mathbb{A}$ is defined as follows:

- $[\cdot] \in \mathscr{N}_{\mathbb{A}}$

- If $t_{1}, \ldots, t_{m} \in \mathscr{E}_{\mathbb{A}}, u \in \mathscr{N}_{\mathbb{A}}$ and $c$ is a constructor of $\mathbb{A}$ with arity $m+1$, then $c t_{1} \ldots t_{i} u t_{i+1} \ldots t_{m} \in \mathscr{N}_{\mathbb{A}}$, for every $i \in\{0, m\}$.

In other words, elements of $\mathscr{N}_{\mathbb{A}}$ are terms with a hole inside them. If $u \in \mathscr{N}_{\mathbb{A}}$ and $t \in \mathscr{E}_{\mathbb{A}}$, then $u[t] \in \mathscr{E}_{\mathbb{A}}$ is obtained by replacing the hole inside $u$ with $t$ in the obvious way. Similarly, if $u, t \in \mathscr{N}_{\mathbb{A}}$, then $u[t]$ will be a context in $\mathscr{N}_{\mathbb{A}} \cdot \mathscr{N}_{\mathscr{A}}$ is defined in the usual way.

The classes $\mathscr{C}_{\mathscr{A}}^{+}$and $\mathscr{C}_{\mathscr{A}}^{-}$of positive and negative type contexts are defined as follows:

- $[\cdot] \in \mathscr{C}_{\mathscr{A}}^{+}$is a positive type context.

- If $L \in \mathscr{C}_{\mathscr{A}}^{+}$and $A$ is a type, then $L \multimap A \in \mathscr{C}_{\mathscr{A}}^{-}$and $A \multimap L \in \mathscr{C}_{\mathscr{A}}^{+}$.

- If $L \in \mathscr{C}_{\mathscr{A}}^{-}$and $A$ is a type, then $L \multimap A \in \mathscr{C}_{\mathscr{A}}^{+}$and $A \multimap L \in \mathscr{C}_{\mathscr{A}}^{-}$.

$\mathscr{C}_{\mathscr{A}}$ is just $\mathscr{C}_{\mathscr{A}}^{+} \cup \mathscr{C}_{\mathscr{A}}^{-}$. A context $L$ is a focus for $A$ if there are a free algebra and a natural number $i$ such that $\mathbb{A}$ and $A \equiv L\left[\mathbb{A}^{i}\right]$. Given an interaction graph $G$, its context semantics is given by a set $T(G)$ of trees. Vertices of trees in $T(G)$ are labelled 

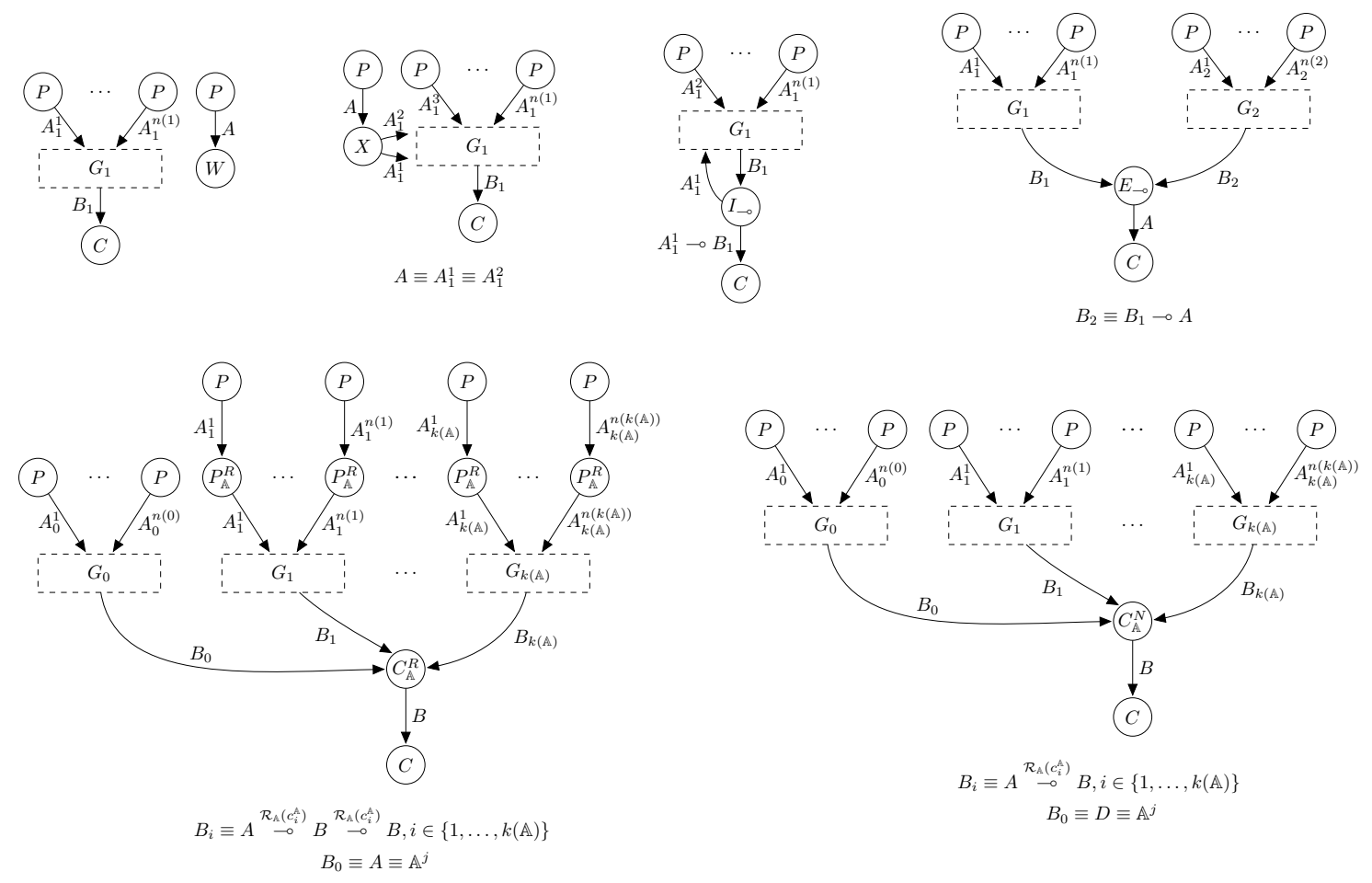

Figure 4. Inductive cases

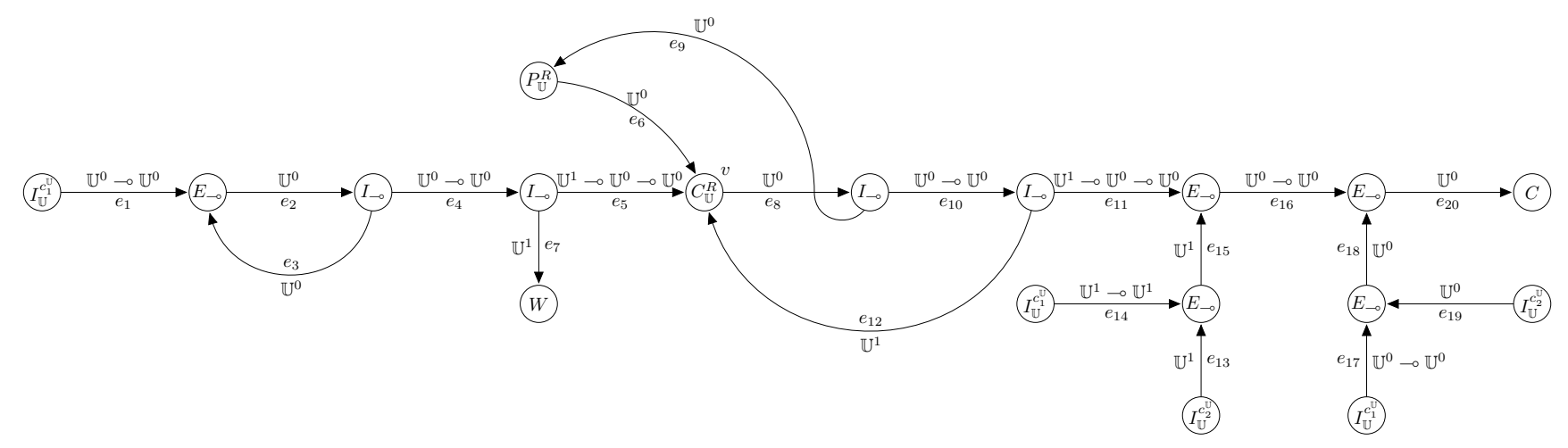

Figure 5. The interaction graph corresponding to a type derivation for UnAdd $\ulcorner 1\urcorner\ulcorner 1\urcorner$ 


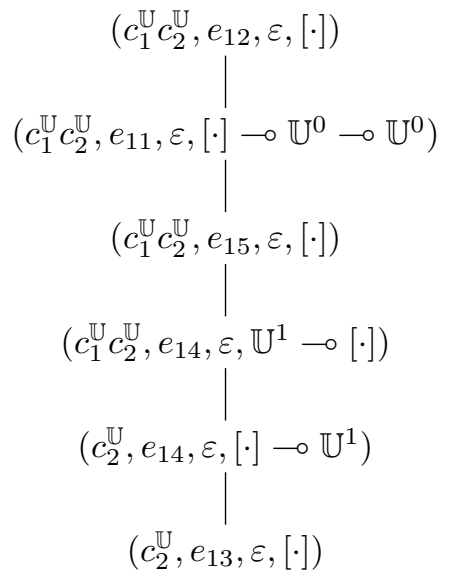

(a)
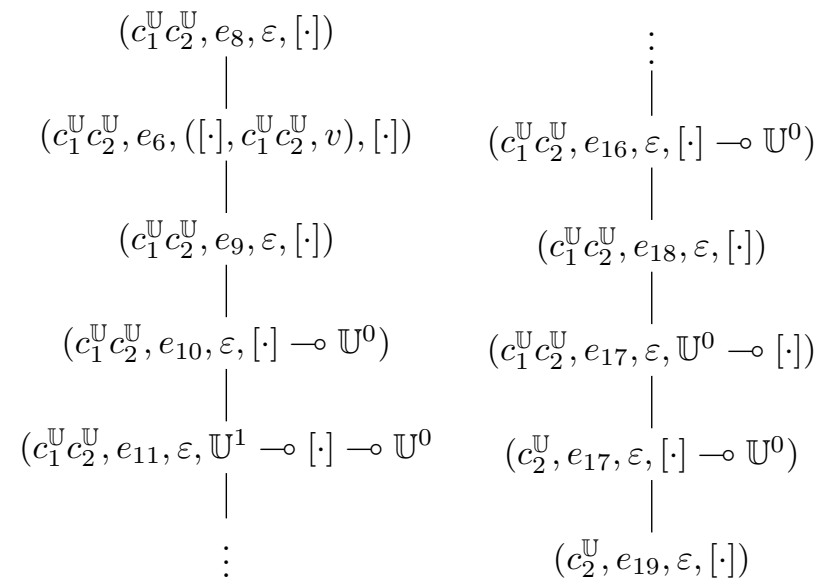

(b)

Figure 6. Examples of trees.

with contexts in $S(G)$, where

$$
\begin{aligned}
S(G) & =\mathscr{E}_{\mathscr{A}} \times E_{G} \times C(G) \times \mathscr{C}_{\mathscr{A}} ; \\
C(G) & =\left(\mathscr{N}_{\mathscr{A}} \times \mathscr{E}_{\mathscr{A}} \times V_{G}\right)^{*} .
\end{aligned}
$$

In other words, elements of $T(G)$ are couples $(l, f)$ where $l \in S(G), f:\{1, \ldots, k\} \rightarrow T(G)$ and $k \in \mathbb{N}$ Elements of $C(G)$ are dubbed stacks. To any tree $T \in T(G)$ we can put in correspondence a term $t=L(T) \in \mathscr{E}_{\mathscr{A}}$ by picking up the first component of the tuple labelling its root.

We will define $T(G)$ by closure conditions. Most of them will be like the following: if $\left(l_{1}, f_{1}\right), \ldots,\left(l_{k}, f_{k}\right) \in T(G)$, then $(l, f) \in T(G)$, where $\forall i \in\{1, \ldots, k\} . f(i)=\left(l_{i}, f_{i}\right)$; These conditions are denoted by writing $l \leftarrow\left(l_{1}, f_{1}\right), \ldots,\left(l_{k}, f_{k}\right)$ or $l \leftarrow l_{1}, \ldots, l_{k}$ if this does not cause ambiguity. More specifically, any closure condition we are going to define will be in one of the following two forms:

- In the form $(t, e, U, L) \leftarrow(t, g, V, M)$. In other words, the root of the newly defined tree will have just one immediate descendant and the term labelling the first component of the root will be the same as the term labelling the first component of its immediate descendant.

- In the form $\left(c t_{1} \ldots t_{n}, e, U, L\right) \leftarrow\left(t_{1}, g_{1}, V_{1}, M_{1}\right), \ldots\left(t_{n}, g_{n}, V_{n}, M_{n}\right)$. In this case, the root of the newly defined tree will have $n$ immediate descendants and the term labelling the first component of the root will be built by applying a constructor to terms appearing as first components of its immediate descendants.

As a consequence, if $t=u[s]$, then there is at least one subtree $S$ of $T$ such that $L(S)=s$ and $S$ somehow corresponds to $u$. We will denote the smallest of such subtrees by $B(T, u, s)$. Observe the root of $B(T, u, s)$ is the last node we find when travelling from the root of $T$ toward its leaves and being guided by $u$.

Formally, $T(G)$ is defined as the smallest set satisfying two families of closure conditions:

- Vertices of $G$ with labels $I_{-}, E_{-}, X I_{\AA}^{c}$ and $C_{\AA}^{N}$ induce closure conditions on $T(G)$. These conditions are detailed in Table[1]

- Every vertex with labels $C_{\mathbb{A}}^{R}$ and $P_{\mathbb{A}}^{R}$ forces $T(G)$ to satisfy more complex closure conditions, as reported in Table 2 In tables 1 and $2 P_{A}$ (respectively, $N_{A}$ ) ranges over positive (respectively, negative) focuses for $A$, while $P_{B}$ (respectively, $N_{B}$ ) ranges over positive (respectively, negative) focuses for $B$. In Figure 6 we report two trees in $T\left(G_{\sigma}\right)$, where $\sigma$ : UnAdd $\ulcorner 1\urcorner\ulcorner 1\urcorner: \mathbb{U}^{0}$.

Branches of trees in $T(G)$ correspond to paths inside $G$, i.e. finite sequences of consecutive edges of $G$. The path corresponding to a branch in $G$ can be retrieved by considering the second component of tuples labelling vertices in the branch. The third and fourth components serve as contexts and are necessary to build the tree in a correct way. Indeed, this way of building trees by traversing paths is reminiscent of token machines in the context of game semantics and geometry of interaction (see [9 [10]). Using this terminology, we can informally describe the components of a tuple as follows: 
Table 1. Closure conditions

\begin{tabular}{|c|c|}
\hline A & $\begin{aligned}\left(t, e, U, P_{A}\right) & \leftarrow\left(t, h, U, P_{A} \multimap B\right) \\
\left(t, h, U, N_{A} \multimap B\right) & \leftarrow\left(t, e, U, N_{A}\right) \\
\left(t, h, U, A \multimap P_{B}\right) & \leftarrow\left(t, g, U, P_{B}\right) \\
\left(t, g, U, N_{B}\right) & \leftarrow\left(t, h, U, A \multimap N_{B}\right)\end{aligned}$ \\
\hline & $\begin{aligned}\left(t, e, U, P_{A} \multimap B\right) & \leftarrow\left(t, g, U, P_{A}\right) \\
\left(t, g, U, N_{A}\right) & \leftarrow\left(t, e, U, N_{A} \multimap B\right) \\
\left(t, e, U, A \multimap N_{B}\right) & \leftarrow\left(t, h, U, N_{B}\right) \\
\left(t, h, U, P_{B}\right) & \leftarrow\left(t, e, U, A \multimap P_{B}\right)\end{aligned}$ \\
\hline$e$ & $\begin{array}{l}(t, e, U,[\cdot]) \leftarrow(t, h, U,[\cdot]) \\
(t, g, U,[\cdot]) \leftarrow(t, h, U,[\cdot])\end{array}$ \\
\hline$\sqrt{\mathbb{A}}$ & $\begin{array}{l}\text { Let } T=\left(\left(u[t], \rho_{G}(v), U,[\cdot]\right), f\right) \in T(G) \text { and } \theta_{G}(e)=v \text {. Then } \\
\qquad(c, e,(u, t, v) U,[\cdot]) \leftarrow \\
\text { If } \theta_{G}(e) \text { is undefined, then } \\
\qquad(c, e, \varepsilon,[\cdot]) \leftarrow\end{array}$ \\
\hline$e \underset{\mathbb{A}}{\stackrel{n \geq 1}{\longrightarrow} \mathbb{A}}$ & $\begin{array}{c}\left(c t_{1} \ldots t_{\mathcal{R}_{\mathbb{A}}(c)}, e, U, \mathbb{A} \stackrel{n}{\multimap}[\cdot]\right) \leftarrow \\
\left(t_{1}, e, U, \mathbb{A} \multimap[\cdot] \multimap(\mathbb{A} \multimap \mathbb{A} \multimap)\right. \\
\vdots \\
\left(t_{\mathcal{R}_{\mathbb{A}}(c)}, e, U, \mathbb{A} \stackrel{n-1}{\multimap}[\cdot] \multimap(\mathbb{A} \multimap \mathbb{A})\right)\end{array}$ \\
\hline $\begin{aligned} B_{i} \equiv \mathbb{A} \stackrel{n_{i}}{\circ} A, i \geq 1 \\
n_{i}= \\
\mathcal{R}_{\mathbb{A}}\left(c_{i}^{\mathbb{A}}\right), i \geq 1 \\
\\
B_{0} \equiv \mathbb{A}\end{aligned}$ & $\begin{aligned} & \text { Let } T=\left(\left(c_{i} t_{1} \ldots t_{n_{i}}, e_{0}, U,[\cdot]\right), f\right) \in T(G) \text { and } S_{j}= \\
& B\left(T, c_{i} t_{1} \ldots t_{j-1}[\cdot] t_{j+1} \ldots t_{n_{i}}, t_{j}\right) . \text { Then } \\
&\left(t, g, U, P_{A}\right) \leftarrow\left(t, e_{i}, U, \mathbb{A} \stackrel{n_{i}}{\longrightarrow} P_{A}\right) \\
&\left(t, e_{i}, U, \mathbb{A} \stackrel{n_{i}}{\circ} N_{A}\right) \leftarrow\left(t, g, U, N_{A}\right) \\
&\left(t_{i}, e_{j}, U, \mathbb{A} \stackrel{n_{i}-j}{\circ}[\cdot] \multimap \mathbb{A} \stackrel{j}{\circ-1} A\right) \leftarrow S_{j}\end{aligned}$ \\
\hline
\end{tabular}


Table 2. Closure conditions

\begin{tabular}{|c|c|}
\hline 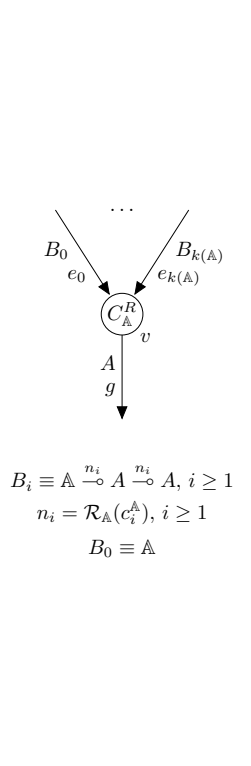 & 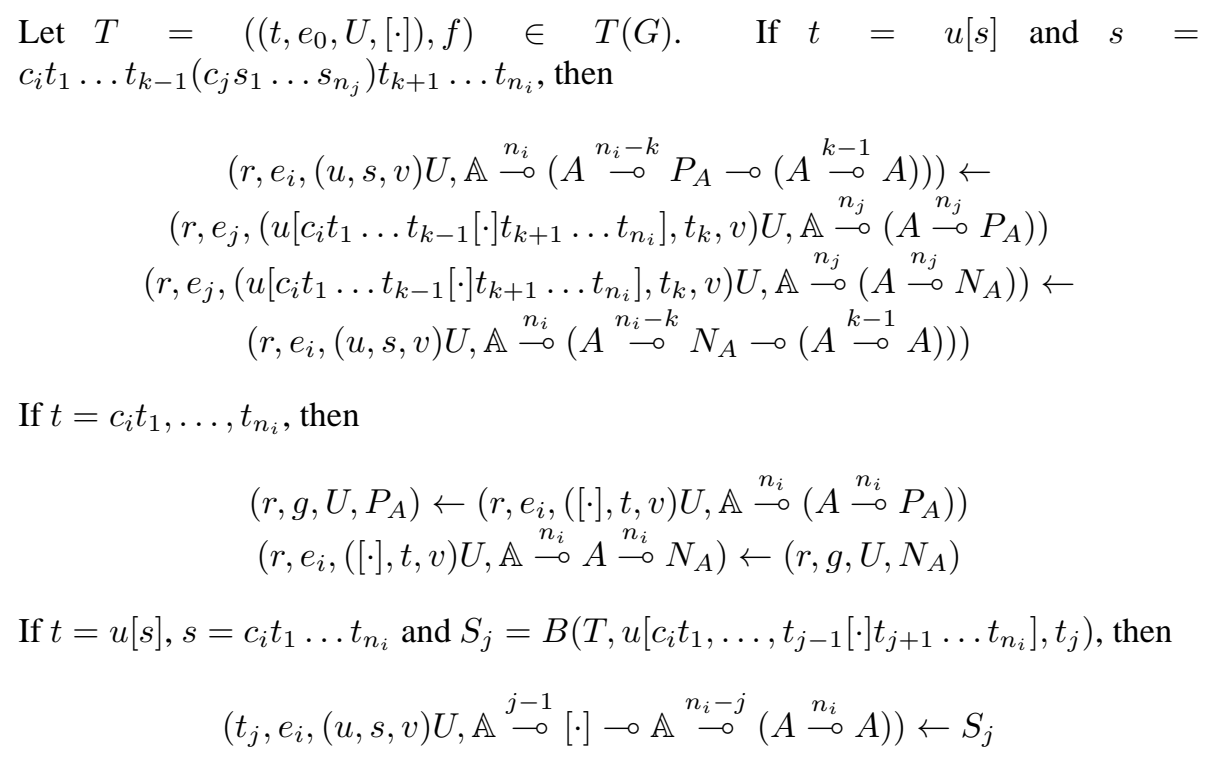 \\
\hline $\begin{array}{c}e \\
\mathbb{A} \downarrow \\
\left(P_{\mathbb{A}}^{R}\right) \\
g \\
\mathbb{A}\end{array}$ & $\begin{array}{r}\text { Let } T=\left(\left(u[s], \rho_{G}(v), U,[\cdot]\right), f\right) \in T(G) \text { and } \theta_{G}(g)=v \text {. Then } \\
(t, g,(u, s, v) U,[\cdot]) \leftarrow(t, e, U,[\cdot])\end{array}$ \\
\hline
\end{tabular}

- The first component is a value carried by the token; it is modified when crossing a node labelled with $I_{\AA}^{c}$;

- the third one is a stack and can only be changed by traversing a node labelled with $C_{\AA}^{R}$ or $P_{\AA}^{R}$;

- the fourth component is a type context guiding the travel of the token. As we are going to show in the following, the fourth component is always a focus for the type labelling the current edge (which can be found in the second component).

Some observations about the closure conditions in tables 1 and 2 are now in order:

- The only way of proving a one-node tree to be in $T(G)$ consists in applying the closure condition induced by a vertex $w$ labelled with $I_{\mathbb{A}}^{c}$, where $\mathcal{R}_{\mathbb{A}}(c)=0$. Notice that, if $\theta_{G}(w)$ is defined (i.e. $w$ is inside a box), we must check the existence of another (potentially big) tree $T$. Similarly when we want to "enter" a box by traversing a vertex $w$ labelled with $P_{\mathbb{A}}^{R}$.

- Closure conditions induced by vertices labelled with $C_{\mathbb{A}}^{R}$ are quite complicated. Consider one such vertex $w$. First of all, a preliminary condition to be checked is the existence of a node $T$ such that the second component of the tuple labelling the root of $T$ is the recursive premise of $w$. The existence of $T$ certifies that exactly $|L(T)|$ copies of the box under consideration will be produced during reduction, each of them corresponding to a tuple $(u, s, w)$ where $u[s]=L(T)$. The vertex $w$ induce five distinct closure rules. The first two rules correspond to paths that come from the interior of the box under consideration and stay inside the same box: we go from one copy of the box to another one and, accordingly, the leftmost element of the underlying stack is changed. The third and fourth rules correspond to paths that enter or exit the box from its conclusion: an element of $C(G)$ is either popped from the underlying stack (when exiting the box) or pushed into it (when enterint the box). The last rule is definitely the trickiest one. First of all, remember that $L(T)$ represents an argument to the recursion corresponding to $w$. If we look at the reduction rule for recursive redexes, we immediately realize that subterms of this argument should be passed to the bodies of the recursion itself. Now, suppose we want to build a new tree in the context semantics by extending $T$ itself. In other, word, suppose we want to proceed with the paths corresponding to $T$. Intuitively, those paths should proceed inside the box. However, we cannot extend $T$ itself, but subtrees of it. This is the reason why we extend $S_{j}$ and not $T$ itself in the last rule. 
- Closure conditions induced by vertices labelled with $C_{\mathbb{A}}^{N}$ can be seen as slight simplifications on those induce by vertices labelled with $C_{\mathbb{A}}^{R}$. Here there are no box, we do not modify the underlying stack and, accordingly, there are no rules like the first two rules induced by $C_{\mathbb{A}}^{R}$.

$T(G)$ has been defined as the smallest set satisfying certain closure conditions. This implies it will only contain finite trees. Moreover, it can be endowed with an induction principle, which does not coincide with the trivial one. For example, the first of the two trees reported in Figure 6 is smaller (as an element of $T(G)$ ) than the second one, even if it is not a subtree of it. Saying it another way, proving properties about trees $T \in T(G)$ we can induce on the structure of the proof that $T$ is an element of $T(G)$ rather than inducing on the structure of $T$ as a tree. This induction principle turns out to be very powerful and will be extensively used in the following.

If $T \in T(G)$, we will denote by $U(T)$ the set containing all the elements of $C(G)$ which appear as third components of labels in $T$. The elements $U(T)$ are the legal stacks for $T$. Stacks in $U(T)$ have a very constrained structure. In particular, all vertices found (as third components of tuples) in a legal stack are labelled with $C_{\AA}^{R}$ and are precisely the vertices of this type which lie at boundaries of boxes in which the current edge (the second component of the tuple labelling the root of $T$ ) is contained. Moreover, if a term context $u$ and a term $s$ are found (as first and second components of tuples) in a legal stack, then there must be a certain tree $S$ such that $L(S)=u[s]$. More precisely:

Lemma 1 (Legal Stack Structure) For every $T \in T_{G}$, for every $\left(t, e,\left(u_{1}, t_{1}, v_{1}\right) \ldots\left(u_{k}, t_{k}, v_{k}\right), L\right)$ appearing as a label of a vertex of $T$ :

- for every $i \in\{1, \ldots, k\}$ there is $f$ such that $\left(\left(u_{i}\left[t_{i}\right], \rho_{G}\left(v_{i}\right), U_{i},[\cdot]\right), f\right) \in T_{G}$, where $U_{i}=\left(u_{i+1}, t_{i+1}, v_{i+1}\right) \ldots\left(u_{k}, t_{k}, v_{k}\right)$;

- for every $i \in\{1, \ldots, k-1\}, \theta_{G}\left(v_{i}\right)=v_{i+1}$;

- $\theta_{G}\left(v_{k}\right)$ is undefined.

Moreover, $k=0$ iff $\theta_{G}(e)$ is undefined and if $k \geq 1$, then $\theta_{G}(e)=v_{1}$.

Proof. By a straightforward induction on the proof that $T \in T(G)$.

\subsection{Completeness}

This section is devoted to proving the completeness of algebraic context semantics as a way to get the algebraic potential size of a term:

Theorem 1 (Completeness) If $\pi: \Gamma \vdash_{\mathrm{H}(\mathrm{A})} M: A, M \sim^{*} N$ and is the argument of a redex in $N$, then there is $T \in T(G)$ such that $L(T)=t$.

Two lemmas will suffice for proving Theorem 1 On one side, arguments of redexes inside a term $M$ can be retrieved in the context semantics of $M$ :

Lemma 2 (Adequacy) If $\pi: \Gamma \vdash_{\mathrm{H}(\mathrm{A})} M: A$ and $M$ contains a redex with argument $t$, then there is $T \in T\left(G_{\pi}\right)$ such that $L(T)=t$.

Proof. First of all, we can observe that there must be a subderivation $\xi$ of $\pi$ such that $\xi: \Delta \vdash t: \mathbb{A}^{i}$. Moreover, the path from the root of $\pi$ to the root of $\xi$ does not cross any instance of rule $E_{-\circ}^{R}$. We can prove that there is $e \in E_{G_{\xi}}$ such that $(t, e, \varepsilon,[\cdot]) \in T\left(G_{\xi}\right)$ by induction on the structure of $\xi$ (with some effort if $\mathbb{A}$ is not a word algebra). The thesis follows once we observe that $G_{\xi}$ is a subgraph of $G_{\pi}, \theta_{G_{\xi}}(e)$ is always undefined and $\theta_{G_{\pi}}(e)$ is undefined whenever $e$ is part of the subgraph of $G_{\pi}$ corresponding to $G_{\xi}$.

This, however, does not suffice. Context semantics must also reflect arguments that will eventually appear during normalization:

Lemma 3 (Backward Preservation) If $\pi: \Gamma \vdash_{\mathrm{H}(\mathrm{A})} M: A$ and $M \leadsto N$, there is $\xi: \Gamma \vdash N:$ such that whenever $T \in T\left(G_{\xi}\right)$, there is $S \in T\left(G_{\pi}\right)$ with $L(S)=L(T)$.

Proof. First of all we will prove the following lemma: if $\pi: \Gamma, x: A \vdash M: B$ and $\xi: \Delta \vdash V: A$, then the interaction graph $G_{\sigma}$, where $\sigma: \Gamma, \Delta \vdash M\{V / x\}: B$ can be obtained by plugging $G_{\xi}$ into the premise of $G_{\pi}$ corresponding to $x$ and applying one or more rewriting steps as those in Figure 7 a). This lemma can be proved by an induction on the structure of $\pi$. 
Now, suppose $\pi: \Gamma \vdash M: A$ and $M \leadsto N$ by firing a beta redex. Then, a type derivation $\xi: \Gamma \vdash N: A$ can be obtained from $\pi$ applying one rewriting step as that in Figure 7 b) and one or more rewriting steps as those in Figure 7 a). One can verify that, for every rewriting step in Figure 7 if $H$ is obtained from $G$ applying the rewriting step and $T \in T(H)$, then there is $S \in T(G)$ such that $L(S)=L(T)$.

We now prove the same for conditional and recursive redexes. To keep the proof simple, we assume to deal with conditionals and recursion on the algebra $\mathbb{U}$. Suppose, $\pi: \Gamma \vdash M: A$ and $M \leadsto N$ by firing a recursive redex $c_{1}^{\mathbb{U}} t\left\langle\left\langle M_{1}, M_{2}\right\rangle\right\rangle$. Then there is a type derivation $\xi: \Gamma \vdash N: A$ such that $G_{\xi}$ can be obtained from $G_{\pi}$ by rewriting as in Figure 8 a). We can define a partial function

$$
\varphi: E_{G_{\xi}} \times C\left(G_{\xi}\right) \times \mathscr{C}_{\mathscr{A}} \rightarrow E_{G_{\pi}} \times C\left(G_{\pi}\right) \times \mathscr{C}_{\mathscr{A}}
$$

in such a way that if $((t, e, U, L), f) \in T\left(G_{\xi}\right)$, then there is $((t, \varphi(e, U, L)), h) \in T\left(G_{\pi}\right)$. In definint $\varphi$, we will take advantage of Lemma 1 For example, we can assume $U=\varepsilon$ whenever $\left(t, e_{2}, U, L\right)$ appear as a label of any $T \in T\left(G_{\xi}\right)$. Indeed, we cannot fire any recursive redex "inside a box", because the reduction relation $\leadsto$ would forbid it.

- The function $\varphi$ acts as the identity on triples $(e, U, L)$ where $e$ lies outside the portion of $G_{\xi}$ affected by rewriting.

- Observe there are two copies of $G(t)$ in $G_{\xi}$; if $e$ is an edge of one of these two copies, then $\varphi(e, U, L)$ will be $(g, U, L)$, where $g$ is the edge corresponding to $e$ in $G\left(c_{1}^{\mathbb{U}} t\right)$.

- Observe there are two copies of $G\left(M_{1}\right)$ in $G_{\xi}$, the leftmost one inside a box whose premise is $w$, and the rightmost outside it; if $e$ is an edge of the rightmost of these two copies, then $\varphi(e, U, L)$ will be $\left(g, U\left([\cdot], c_{1}^{\mathbb{U}} t, v\right), L\right)$, where $g$ is the edge corresponding to $e$ in $G\left(M_{1}\right)$; if $e$ is an edge of the leftmost of these two copies, then $\varphi(e, U(u, s, w), L)$ will be $\left(g, U\left(c_{1}^{\mathbb{U}} u, s, v\right), L\right)$.

- In $G_{\xi}$ there is just one copy of $G\left(M_{2}\right)$; if $e$ is an edge of this copy of $G\left(M_{2}\right)$, then $\varphi(e, U(u, s, w), L)$ will be $\left(g, U\left(c_{1}^{\mathbb{U}} u, s, v\right), L\right)$.

- The following equations hold:

$$
\begin{aligned}
\varphi\left(e_{1}^{i}, \varepsilon, L\right) & =\left(g_{1}^{i}, \varepsilon, L\right) \\
\varphi\left(e_{2}, \varepsilon, L\right) & =\left(g_{2},\left([\cdot], c_{1}^{\mathbb{U}} t, v\right), \mathbb{U} \multimap L \multimap A\right) \\
\varphi\left(e_{3}, \varepsilon, A \multimap L\right) & =\left(g_{3}, \varepsilon, L\right) \\
\varphi\left(e_{3}, \varepsilon, L \multimap A\right) & =\left(g_{2},\left([\cdot], c_{1}^{\mathbb{U}} t, v\right), \mathbb{U} \multimap L \multimap A\right)
\end{aligned}
$$

We can prove that if $T=((r, e, \varepsilon, L), f) \in T\left(G_{\xi}\right)$, then there is $((r, \varphi(e, \varepsilon, L)), h) \in T\left(G_{\pi}\right)$ by induction on $T$. Let us just analyze some of the most interesting cases:

- Suppose there is a tree $T \in T\left(G_{\xi}\right)$ whose root is labelled with $\left(r, e_{4}^{i}, \varepsilon,[\cdot]\right)$. By applying the closure rule induced by vertices labelled with $X$, we can extend $T$ to a tree whose root is labelled with $\left(r, e_{1}^{i}, \varepsilon,[\cdot]\right)$. By the induction hypothesis applied to $T$, there is a tree in $T\left(G_{\pi}\right)$ whose root is labelled with $\left(r, \varphi\left(e_{4}^{i}, \varepsilon,[\cdot]\right)\right)=\left(r, g_{1}^{i}, \varepsilon,[\cdot]\right)$. But observe that $\varphi\left(e_{1}^{i}, \varepsilon,[\cdot]\right)=\left(g_{1}^{i}, \varepsilon,[\cdot]\right)$.

- Suppose there is a tree $T \in T\left(G_{\xi}\right)$ whose root is labelled with $\left(r, e_{4}^{i}, \varepsilon,[\cdot]\right)$. By applying the closure rule induced by vertices labelled with $X$, we can extend $T$ to a tree $S$ whose root is labelled with $\left(r, e_{5}^{i}, \varepsilon,[\cdot]\right)$. By the induction hypothesis applied to $T$, there is a tree $S \in T\left(G_{\pi}\right)$ whose root is labelled with $\left(r, \varphi\left(e_{4}^{i}, \varepsilon,[\cdot]\right)\right)=\left(r, g_{1}^{i}, \varepsilon,[\cdot]\right)$. By applying the closure rule induced by vertices labelled with $P_{\mathbb{U}}^{R}$, we can extend $S$ to a tree in $T\left(G_{\pi}\right)$ whose root is labelled with $\left(r, g_{4}^{i},\left([\cdot], c_{1}^{\mathbb{U}} t, v\right),[\cdot]\right)$ But observe that $\varphi\left(e_{5}^{i}, \varepsilon,[\cdot]\right)=\left(g_{4}^{i},\left([\cdot], c_{1}^{\mathbb{U}} t, v\right),[\cdot]\right)$, because $e_{5}^{i}$ is part of the rightmost copy of $G\left(M_{1}\right)$.

- Suppose there is a tree $T \in T\left(G_{\xi}\right)$ whose root is labelled with $\left(r, e_{11}, \varepsilon, L\right)$ and $L$ is a negative type context. By applying the closure rule induced by vertices labelled with $E_{-}$, we can extend $T$ to a tree whose root is labelled with $\left(r, e_{3}, \varepsilon, A \multimap\right.$ $L)$ and, by applying again the same closure rule, we can obtain a tree whose root is labelled with $\left(r, e_{6}, \varepsilon, \mathbb{U} \multimap A \multimap L\right)$. By the induction hypothesis applied to $T$, there is a tree $S \in T\left(G_{\pi}\right)$ whose root is labelled with $\left(r, \varphi\left(e_{11}, \varepsilon, L\right)\right)=$ $\left(r, g_{3}, \varepsilon, L\right)$. Observe that $\varphi\left(e_{3}, \varepsilon, A \multimap L\right)=\left(g_{3}, \varepsilon, L\right)$. By applying the closure rule induced by vertices labelled with $C_{\mathbb{U}}^{R}$, we can extend $S$ to a tree in $T\left(G_{\pi}\right)$ whose root is labelled with $\left(r, g_{2},\left([\cdot], c_{1}^{\mathbb{U}} t, v\right), \mathbb{U} \multimap A \multimap L\right)$. But observe that $\varphi\left(e_{6}, \varepsilon, \mathbb{U} \multimap A \multimap L\right)=\left(g_{2},\left([\cdot], c_{1}^{\mathbb{U}} t, v\right), \mathbb{U} \multimap A \multimap L\right)$, because $e_{6}$ is part of the rightmost copy of $G\left(M_{1}\right)$.

- Suppose there is a tree $T \in T\left(G_{\xi}\right)$ whose root is labelled with $\left(r, e_{6}, \varepsilon, \mathbb{U} \multimap L \multimap A\right)$ and $L$ is a negative type context. By applying the closure rule induced by vertices labelled with $E_{-\circ}$, we can extend $T$ to a tree whose root is labelled with $\left(r, e_{3}, \varepsilon, L \multimap A\right)$ and, by applying another closure rule induced by the same vertex, we can obtain a tree whose root is labelled with $\left(r, e_{2}, \varepsilon, L\right)$. By the induction hypothesis applied to $T$, there is a tree $S \in T\left(G_{\pi}\right)$ whose root is labelled with $\left(r, \varphi\left(e_{6}, \varepsilon, \mathbb{U} \multimap L \multimap A\right)\right)=\left(r, g_{2},\left([\cdot], c_{1}^{\mathbb{U}} t, v\right), \mathbb{U} \multimap L \multimap A\right)$. Observe that $\varphi\left(e_{3}, \varepsilon, L \multimap A\right)=\left(g_{2},\left([\cdot], c_{1}^{\mathbb{U}} t, v\right), \mathbb{U} \multimap\right.$ $L \multimap A)$ and $\varphi\left(e_{2}, \varepsilon, L\right)=\left(g_{2},\left([\cdot], c_{1}^{\mathbb{U}} t, v\right), \mathbb{U} \multimap L \multimap A\right)$. 
This shows that the thesis holds for recursive redexes in the form $c_{1}^{\mathbb{U}} t\left\langle\left\langle M_{1}, M_{2}\right\rangle\right\rangle$. Similar arguments hold for redexes in the form $c_{2}^{\mathbb{U}}\left\langle\left\langle M_{1}, M_{2}\right\rangle\right\rangle, c_{1}^{\mathbb{U}} t\left\{\left\{M_{1}, M_{2}\right\}\right\}, c_{2}^{\mathbb{U}}\left\{\left\{M_{1}, M_{2}\right\}\right\}$ (see Figure 8]b), Figure 8 (c) and Figure 8]d), respectively).

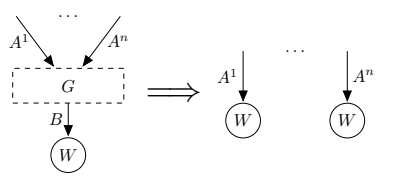

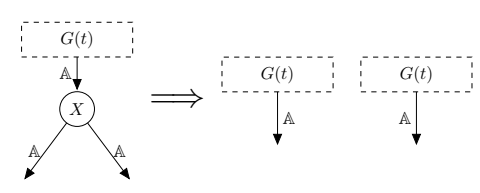

(a)

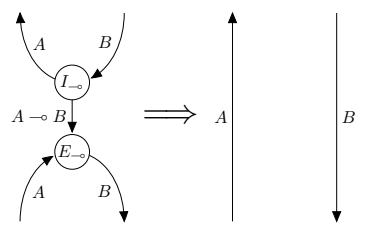

(b)

Figure 7. Graph transformation produced by firing a beta-redex.

Summing up, any possible algebraic term appearing in any possible reduct of a typable term $M$ can be found in the context semantics of the interaction graph for a type derivation for $M$. This proves Theorem 1

\section{On the Complexity of Normalization}

In this section, we will give some bounds on the time needed to normalize terms in subsystems $H(A), R H(A)$ and $R H(W)$. Our strategy consists in studying how constraints like linearity and ramification induce bounds on $|L(T)|$, where $T$ is any tree built up from the context semantics. These bounds, by Theorem 1 and Proposition 2 translate into bounds on normalization time (modulo appropriate polynomials). Noticeably, many properties of the context semantics which are very useful in studying $|L(T)|$ are true for all of the above subsystems and can be proved just once. These are precisely the properties that that will be proved in the first part of this section.

First of all we observe that, by definition, every subtree of $T \in T(G)$ is itself a tree in $T(G)$. Moreover, a uniqueness property can be proved:

Proposition 3 (Uniqueness) For every interaction graph $G$, for every e $\in E_{G}, U \in C(G)$ and $L \in \mathscr{C}_{\mathscr{A}}$, there is at most one tree $T \in T(G)$ such that $T=((t, e, U, L), f)$.

Proof. We can show the following: if $((t, e, U, L), f) \in T(G)$, then there cannot be $((s, e, U, L), g) \in T(G)$, where $s \neq t$ or $f \neq g$. We can prove this by an induction on the structure of the proof that $((t, e, U, L), f) \in T(G)$. First of all, observe that $L$ and $e$ uniquely determine the last closure rule used to prove that $((t, e, U, L), f) \in T(G)$. In particular, if $e=(v, w)$ and $L$ is positive, then it is one induced by $v$, otherwise it is one induced by $w$. At this point, however, one can easily see that the domains of $f$ and $g$ must be the same. So, there must be some $i$ such that $f(i)$ and $g(i)$ are different, but with the same label for the root. This, however, would contraddict the inductive hypothesis.

The previous result implies the following: every triple $(e, U, L) \in E_{G} \times C(G) \times \mathscr{C}_{\mathscr{A}}$ can appear at most once in any branch of any $T \in T(G)$. As a consequence, any $T \in T(G)$ (and, more importantly, any $t$ such that $t=L(T)$ for some $T \in T(G)$ ) cannot be too big compared to $|C(G)|$ and $|G|$. But, in turn, the structure of relevant elements of $C(G)$ is very contrived. Indeed, Lemma 1 implies the length of any stack in $U(T)$ where $T \in T\left(G_{\pi}\right)$ cannot be bigger than the recursion depth $R(\pi)$ of $\pi$ : the length of $U$ is equal to the "depth" of $e$ whenever $(t, e, U, L)$ appears as a label in $T$.

Along a path, the fourth component of the underlying tuple can change, but there is something which stays invariant:

Lemma 4 For every $T \in T(G)$ there is a type $\mathbb{A}^{i}$ such that for every $(t, e, U, L)$ appearing as a label of a vertex of $T$, $\beta_{G}(e)=L\left[\mathbb{A}^{i}\right]$. We will say $T$ is guided by $\mathbb{A}^{i}$.

Proof. By a straigthforward induction on the proof that $T \in T(G)$.

The previous lemmas shed some light on the combinatorial properties of tuples $(t, e, U, L) \in S\left(G_{\pi}\right)$ labelling vertices of trees in $T\left(G_{\pi}\right)$. This is enough to prove $|L(T)|$ to be exponentially related to the cardinality of $U(T)$ : 


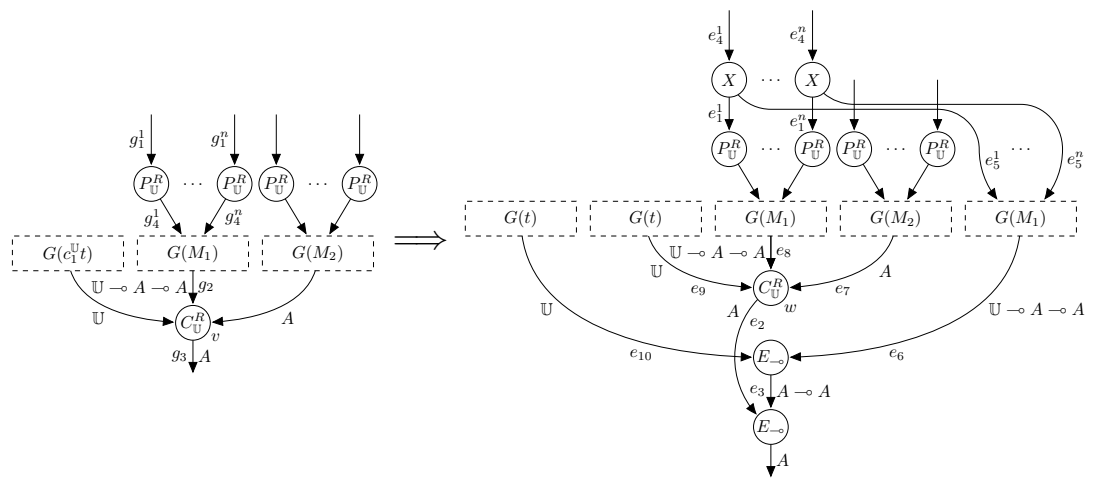

(a)

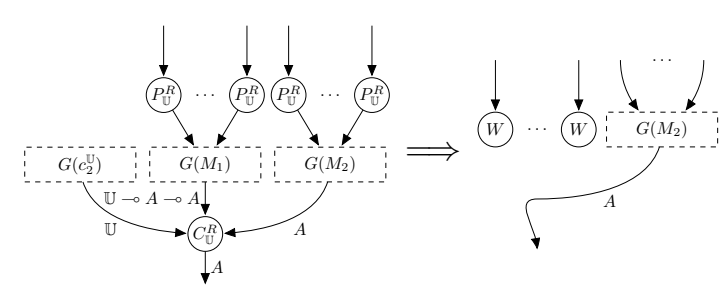

(b)

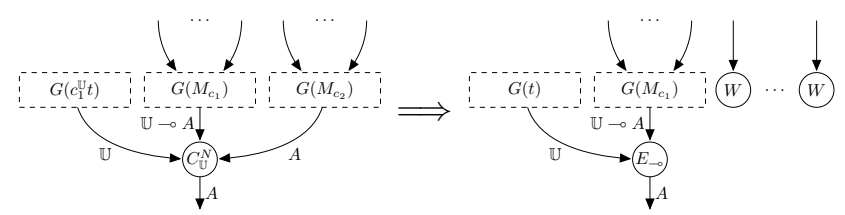

(c)

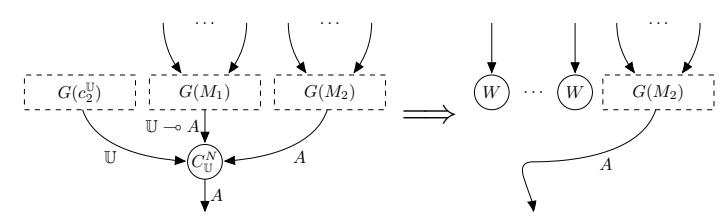

(d)

Figure 8. The graph transformations induced by firing a recursive or conditional redex.

Proposition 4 Suppose $\pi: \Gamma \vdash_{\mathrm{H}(\mathrm{A})} M:$ A and $T \in T\left(G_{\pi}\right)$. Then $|L(T)| \leq \mathscr{K}_{\mathscr{A}}^{\left|G_{\pi}\right||U(T)|}$.

Proof. First of all, we observe that whenever $(t, e, U, L)$ labels a vertex $v$ of $T$ and $(s, f, V, M)$ labels one child of $v$, then either $s=t$ or $t=c s_{1} \ldots s_{k}$ and $L=\mathbb{A} \stackrel{k}{\circ}[\cdot]$. The thesis follows from lemmas 1 and 4

This will lead to prove primitive recursive bounds for $\mathrm{H}(\mathrm{A})$ and elementary bounds for $\mathrm{RH}(\mathrm{A})$. However, we cannot expect to prove any polynomial bound from Proposition 4 In the case of $\mathrm{RH}(\mathrm{W})$, a stronger version of Proposition 4 can be proved by exploiting ramification.

Proposition 5 Suppose $\pi: \Gamma \vdash_{\mathrm{RH}(\mathrm{W})} M: A$ and $T \in T\left(G_{\pi}\right)$. Then $|L(T)| \leq\left|G_{\pi}\right||U(T)|$. 
Proof. First of all, we prove the following lemma: for every $T \in T\left(G_{\pi}\right)$, if $T$ is guided by $\mathbb{A}^{i}$ and $\mathbb{A}$ is a word algebra, there are at most one tree $((t, e, U, L), f) \in T\left(G_{\pi}\right)$ and one integer $i \in \mathbb{N}$ such that $f(i)=T$. To prove the lemma, suppose $((t, e, U, L), f),((s, g, V, M), h) \in T\left(G_{\pi}\right)$ and $f(i)=g(j)=T . T$ uniquely determines the closure condition used to prove that both $((t, e, U, L), f),((s, g, V, M), h) \in T\left(G_{\pi}\right)$, which must be the same because those induced by typing rule $X$ are forbidden. But by inspecting all the closure rules, we can conclude that $s=t, e=g, U=V, L=M$ and $f(n)=g(n)$ for every $n$. Then, we can proceed exactly as in Proposition 4

Notice how the elementary bound of Proposition 4has become a polynomial bound in Proposition [5] Quite surprisingly, this phase transition happens as soon as the class of types on which we allow contraction is restricted from A to W.

\section{1 $H(A)$ and Primitive Recursion}

Given an interaction graph $G$, we now need to define subclasses $T(\mathcal{U})$ of $T(G)$ for any subset $\mathcal{U}$ of $C(G)$. In principle, we would like $U(T)$ to be a subset of $\mathcal{U}$ whenever $T \in T(\mathcal{U})$. However, this is too strong a constraint, since we should allow $U(T)$ to contain extensions of stacks in $\mathcal{U}$, the extensions being obtained themselves in this constrained way. The following definition captures the above intuition. Let $G$ be an interaction graph and $\mathcal{U} \subseteq C(G)$. A tree $T \in T(G)$ is said to be generated by $\mathcal{U}$ iff for every $U \in U(T)$ :

- either $U \in \mathcal{U}$,

- or $U=\left(u_{1}, t_{1}, v_{1}\right) \ldots\left(u_{k}, t_{k}, v_{k}\right) V$ where $V$ is itself in $\mathcal{U}$ and has maximal length (between all the elements of $\mathcal{U}$. Moreover, for every $i \in\{1, \ldots, k\}$, the tree $\left(\left(u_{i}\left[t_{i}\right], \rho_{G}\left(v_{i}\right),\left(u_{i+1}, t_{i+1}, v_{i+1}\right) \ldots\left(u_{k}, t_{k}, v_{k}\right) V,[\cdot]\right), f\right)$ must be itself generated by $\mathcal{U}$.

The set of all trees generated by $\mathcal{U}$ will be denoted by $T(\mathcal{U})$. This definition is well-posed because of the induction principles on $T(G)$. Indeed, we require some trees $T_{1}, \ldots, T_{n}$ to be in $T(\mathcal{U})$ when defining conditions on $T$ being an element of $T(\mathcal{U})$ itself; however, $T_{1}, \ldots, T_{n}$ are "smaller" than $T$. Notice that $T$ is not monotone as an operator on subsets of $C(G)$. For example, $T(\{\varepsilon\})=T(G)$, while $T(\{\varepsilon, C\}) \subset T(G)$ whenever $C \notin U(T)$ for any $T \in T(G)$. This is due to the requirement of $V$ having maximal length in the definition above.

Lemma 5 For every $d \in \mathbb{N}$ there is a primitive recursive function $p_{d}: \mathbb{N}^{2} \rightarrow \mathbb{N}$ such that if $\pi: \Gamma \vdash_{\mathrm{H}(\mathrm{A})} M: A, \mathcal{U} \subseteq C\left(G_{\pi}\right)$, the maximal length of elements of $\mathcal{U}$ is $n$, and $T \in T(\mathcal{U})$, then $|L(T)| \leq p_{R(\pi)-n}\left(\left|G_{\pi}\right|,|\mathcal{U}|\right)$.

Proof. We can put

$$
\begin{aligned}
p_{0}(x, y) & =\mathscr{K}_{\mathscr{A}}^{x y} \\
\forall i \geq 1 . h_{i}(x, y, 0) & =\mathscr{K}_{\mathscr{A}}^{x y} \\
\forall i \geq 1 . h_{i}(x, y, z+1) & =h_{i}(x, y, z)+p_{i-1}\left(x, y+h_{i}(x, y, z)\right) \\
\forall i \geq 1 . p_{i}(x, y) & =h_{i}(x, y, x y)
\end{aligned}
$$

Every $p_{i}$ and $h_{i}$ are primitive recursive. Moreover, all these functions are monotone in each of their arguments. We will now prove the thesis by induction on $R(\pi)-n$. If $R(\pi)=n$, then there are elements in $\mathcal{U}$ having length equals to $R(\pi)$. This, by Lemma 1 and Proposition 4 implies that if $T \in T(G)$ is generated by $\mathcal{U}$, then $|L(T)|$ is bounded by $p_{0}(|G|,|\mathcal{U}|)$ since none of the elements of $\mathcal{U}$ having maximal length can be extended into an element of $U(T)$ and, as a consequence, $U(T) \subseteq \mathcal{U}$. Now, let us suppose $R(\pi)-n \geq 1$. Let us define $\mathcal{W} \subseteq C(G)$ as follows

$$
\mathcal{W}=\left\{(u, t, v) U \mid U \in \mathcal{U} \text { has maximal length and }\left(\left(u[t], \rho_{G}(v), U,[\cdot]\right), f\right) \in T(\mathcal{U})\right\}
$$

Clearly, $T(\mathcal{U} \cup \mathcal{W})=T(\mathcal{U})$. Now, consider the sequence $\left(v_{1}, U_{1}\right), \ldots,\left(v_{k}, U_{k}\right)$ of all the pairs $\left(v_{i}, U_{i}\right) \in V_{G} \times \mathcal{U}$ such that $\left(u, t, v_{i}\right) U_{i} \in \mathcal{W}$ for some $u, t$. Obviously, $k \leq|G||\mathcal{U}|$. If $k=0$, then the thesis is trivial, since

$$
\begin{aligned}
|L(T)| & \leq \mathscr{K}_{\mathscr{A}}^{|G||\mathcal{U}|} \\
& =h_{R(\pi)-n}(|G|,|\mathcal{U}|, 0) \\
& \leq h_{R(\pi)-n}(|G|,|\mathcal{U}|,|G||\mathcal{U}|) \\
& =p_{R(\pi)-n}(|G|,|\mathcal{U}|) .
\end{aligned}
$$

From now on, suppose $k \geq 1$. Let $\mathcal{W}_{1}, \ldots, \mathcal{W}_{k} \subseteq \mathcal{W}$ be defined as follows: $\mathcal{W}_{i}=\left\{\left(u, t, v_{j}\right) U_{j} \in \mathcal{W} \mid j \leq i\right\}$. By definition, $\mathcal{W}_{k}=\mathcal{W}$. We can assume, without losing generality, that 
- $T_{1}=\left(\left(t_{1}, \rho_{G}\left(v_{1}\right), U_{1},[\cdot]\right), f_{1}\right)$ only contains elements from $\mathcal{U}$ as part of its labels.

- For every $i \in\{2, \ldots, k\}$, the tree $T_{i}=\left(\left(t_{i}, \rho_{G}\left(v_{i}\right), U_{i},[\cdot]\right), f_{i}\right)$ is generated by $\mathcal{U} \cup \mathcal{W}_{i-1}$.

We can now prove that

$$
\sum_{j=1}^{i+1}\left|t_{j}\right| \leq h_{R(\pi)-n}(|G|,|\mathcal{U}|, i)
$$

by induction on $i$. The tree $T_{1}$ only contains elements of $\mathcal{U}$ as part of its labels and, by Proposition 4

$$
\left|t_{1}\right| \leq \mathscr{K}_{\mathscr{A}}^{|G|\left|U\left(T_{1}\right)\right|} \leq \mathscr{K}_{\mathscr{A}}^{|G||\mathcal{U}|}=h_{R(\pi)-n}(|G|,|\mathcal{U}|, 0) .
$$

If $i \geq 1$, by inductive hypothesis (on $i$ ) we get

$$
\sum_{j=1}^{i}\left|t_{j}\right| \leq h_{R(\pi)-n}(|G|,|\mathcal{U}|, i-1) .
$$

This hields $\left|\mathcal{W}_{i}\right| \leq h_{R(\pi)-n}(|G|,|\mathcal{U}|, i-1)$, because for every every term $t_{j}$ there at most $\left|t_{j}\right|$ triples $\left(u, s, v_{j}\right)$ such that $u[s]=t_{j}$. By induction hypothesis (both on $i$ and $R(\pi)-n$ ), we get

$$
\begin{aligned}
\sum_{j=1}^{i+1}\left|t_{j}\right| & =\sum_{j=1}^{i}\left|t_{j}\right|+\left|t_{i+1}\right| \\
& \leq h_{R(\pi)-n}(|G|,|\mathcal{U}|, i-1)+p_{R(\pi)-n-1}\left(|G|,|\mathcal{U}|+h_{R(\pi)-n}(|G|,|\mathcal{U}|, i-1)\right) \\
& =h_{R(\pi)-n}(|G|,|\mathcal{U}|, i),
\end{aligned}
$$

because $T_{i+1}$ is generatged by $\mathcal{U} \cup \mathcal{W}_{i-1}$. So, $|\mathcal{W}|=\left|\mathcal{W}_{k}\right| \leq h_{R(\pi)-n}(|G|,|\mathcal{U}|, k-1)$. Now, suppose $T \in T(\mathcal{U})=$ $T(\mathcal{U} \cup \mathcal{W})$. Then by inductive hypothesis (on $R(\pi)-n$ )

$$
\begin{aligned}
|L(T)| & \leq p_{R(\pi)-n-1}(|G|,|\mathcal{U} \cup \mathcal{W}|) \\
& =p_{R(\pi)-n-1}(|G|,|\mathcal{U}|+|\mathcal{W}|) \\
& =p_{R(\pi)-n-1}\left(|G|,|\mathcal{U}|+\left|\mathcal{W}_{k}\right|\right) \\
& \leq p_{R(\pi)-n-1}\left(|G|,|\mathcal{U}|+h_{R(\pi)-n}(|G|,|\mathcal{U}|, k-1)\right) \\
& \leq h_{R(\pi)-n}(|G|,|\mathcal{U}|, k) \\
& \leq h_{R(\pi)-n}(|G|,|\mathcal{U}|,|G||\mathcal{U}|) \\
& =p_{R(\pi)-n}(|G|,|\mathcal{U}|)
\end{aligned}
$$

This concludes the proof.

As a corollary, we get:

Theorem 2 For every $d \in \mathbb{N}$, there is a primitive recursive function $p_{d}: \mathbb{N} \rightarrow \mathbb{N}$ such that for every type derivation $\pi: \Gamma \vdash_{\mathbf{H}(\mathbf{A})} M: A$, if $T \in T\left(G_{\pi}\right)$ then $|L(T)| \leq p_{R(\pi)}(|M|)$.

Proof. Trivial, since every tree $T \in T\left(G_{\pi}\right)$ is generated by $\{\varepsilon\}$.

Theorem 2 implies, by Proposition 2 that the time needed to normalize a term $M$ with a type derivation $\pi$ in $\mathrm{H}(\mathrm{A})$ is bounded by a primitive recursive function (just depending on the recursion depth of $\pi$ ) applied to the size of $M$. This, in particular, implies that every function $f: \mathbb{N} \rightarrow \mathbb{N}$ which can be represented in $\mathrm{H}(\mathrm{A})$ must be primitive recursive, because all terms corresponding to calls to $f$ can be typed with bounded-recursion-depth type derivations. This is a leitmotif: elementary bounds for $\mathrm{RH}(\mathrm{A})$ and polynomial bounds for $\mathrm{RH}(\mathrm{W})$ will have the same flavour. Observe how this way of formulating soundness results is necessary in a higher-order setting. Indeed, since bounds are given on the normalization time of any term in the subsystem and the subsystem itself is complete for a complexity class, we cannot hope to prove, say, that any term in $\mathrm{H}(\mathrm{A})$ can be normalized with a fixed, primitive recursive, bound on its size. 


\section{$5.2 \mathrm{RH}(\mathrm{A})$ and Elementary Time}

Now, consider the interpretation of branches of trees in $T(G)$ as paths in $G$ : any such path can enter and exit boxes by traversing vertices labelled with $C_{\mathbb{A}}^{R}$ or $P_{\mathbb{A}}^{R}$. The stack $U$ in the underlying context can change as a result of the traversal. Indeed, $U$ changes only when entering and exiting boxes (other vertices of $G$ leave $U$ unchanged, as can be easily verified). As a consequence, by Proposition 4 entering and exiting boxes is essential to obtain a hyperexponential complexity: if paths induced by a tree $T \in T(G)$ do not enter or exit boxes, $U(T)$ will be a singleton and $L(T)$ will be bounded by a fixed exponential on $|G|$. In general, paths induced by trees can indeed enter or exit boxes. If ramification holds, on the other hand, a path induced by a tree guided by $\mathbb{A}^{i}$ entering into a box whose main premise is labelled by $\mathbb{F}^{j}$ (where $j \leq i$ ), will stay inside the box and the third component of the underlying context will only increase in size. More formally:

Lemma 6 Suppose $\pi$ to be a type derivation satisfying the ramification condition, $S \in T\left(G_{\pi}\right)$ to be guided by $\mathbb{A}^{i},(t, e, U, L)$ to label a vertex $v$ of $S$ and $U=(u, t, w) V$, where $\beta_{G}\left(\rho_{G}(w)\right)=\mathbb{F}^{j}$ and $j \leq i$. Then all the ancestors of $v$ in $S$ are labelled with quadruples $(s, f, W, M)$ where $W=Z U$.

Proof. By a straightforward induction on the structure of $S$. In particular, the only vertices in $G_{\pi}$ whose closure conditions affect the third component of $C(G)$ are those labelled with $P_{\mathbb{G}}^{R}$ and $C_{\mathbb{G}}^{R}$, where $\mathbb{G}$ is any free algebra. The rule induced by a vertex $P_{\mathbb{G}}^{R}$, however, makes the underlying stack bigger (from $U$, it becomes $(u, t, v) U$ ). As a consequence, the statment of the lemma is verified. Now, consider rules induced by $C_{\mathbb{G}}^{R}$ vertices:

- The first four rules cannot be applied under this lemma's hypothesis: by ramification $V\left(B_{0}\right)>V(A)$ but this is in contraddiction with $j \leq i$ from lemma's hypothesis.

- The fifth rule is a bit delicate: $S_{j}$ satisfies the lemma, being it a subtree of a tree $T$ to which we can apply the inductive hypothesis. The rule appens a node whose third component is $(u, s, v) U$, where $U$ is the third component of the tuple labelling the root of $T$. The thesis clearly holds.

This concludes the proof.

This in turn allows to prove a theorem bounding the algebraic potential size of terms in system $\operatorname{RH}(\mathrm{A})$ :

Theorem 3 For every $d, e \in \mathbb{N}$, there are elementary functions $p_{e}^{d}: \mathbb{N} \rightarrow \mathbb{N}$ such that for every type derivation $\pi: \Gamma \vdash_{\mathrm{RH}(\mathrm{A})}$ $M: A$, if $T \in T\left(G_{\pi}\right)$ then $|L(T)| \leq p_{R(\pi)}^{I(\pi)}(|M|)$.

Proof. Consider the following elementary functions:

$$
\begin{aligned}
\forall n, m \in \mathbb{N} \cdot p_{m}^{n} & : \mathbb{N} \rightarrow \mathbb{N} \\
p_{m}^{0}(x) & =\mathscr{K}_{\mathscr{A}}^{x^{2}} \\
p_{m}^{n+1}(x) & =\mathscr{K}_{\mathscr{A}}^{x\left(x \cdot p_{m}^{n}(x)\right)^{m}} .
\end{aligned}
$$

First of all, notice that for every $x, m, n, p_{m}^{n+1}(x) \geq p_{m}^{n}(x)$. We will prove that if $T=((t, e, U, L), f) \in T\left(G_{\pi}\right)$ is guided by $\mathbb{A}^{i}$, then $|t| \leq p_{R(\pi)}^{j}\left(\left|G_{\pi}\right|\right)$, where $j=\max \{I(\pi)-i, 0\}$. We go by induction on $j$.

If $j=0$, then $I(\pi) \leq i$. This implies that $|U(T)| \leq\left|G_{\pi}\right|$, by lemmas 1 and 6 Indeed, by Lemma 6 stacks can only get bigger along paths induced by $T$ and any vertex in $G$ uniquely determines the length of stacks (Lemma1). As a consequence, $|t| \leq \mathscr{K}_{\mathscr{A}}^{\left|G_{\pi}\right|^{2}}=p_{R(\pi)}^{0}\left(\left|G_{\pi}\right|\right)$.

Now, suppose the thesis holds for $j$ and suppose $T=((t, e, U, L), f)$ to be guided by $\mathbb{A}^{i}$, where $I(\pi)-i=j+1$. By Lemma 6 and the induction hypothesis, $|U(T)| \leq\left(\left|G_{\pi}\right| p_{R(\pi)}^{j}\left(\left|G_{\pi}\right|\right)\right)^{R(\pi)}$. Indeed, elements of $U(T)$ are stacks in the form $\left(u_{1}, t_{1}, v_{1}\right) \cdots\left(u_{k}, t_{k}, v_{k}\right)$ where $k \leq R(\pi)$ and, for every $l \in\{1, \ldots, k\}$ :

- Either $\beta_{G}\left(\rho_{G}\left(v_{l}\right)\right)=\mathbb{F}^{h}$, where $h \leq i$ and $T$ uniquely determines $u_{l}$ and $t_{l}$ due to Lemma 6 ,

- $\operatorname{or} \beta_{G}\left(\rho_{g}\left(v_{l}\right)\right)=\mathbb{F}^{h}$ where $h>l,\left(u_{l+1}, t_{l+1}, v_{l+1}\right) \cdots\left(u_{k}, t_{k}, v_{k}\right)$ and $v_{l}$ uniquely determines $u_{l}\left[t_{l}\right]$ and $\left|u_{l}\left[t_{l}\right]\right| \leq$ $p_{R(\pi)}^{j}\left(\left|G_{\pi}\right|\right)$ by the inductive hypothesis.

As a consequence,

$$
|t| \leq \mathscr{K}_{\mathscr{A}}^{\left|G_{\pi}\right|\left(\left|G_{\pi}\right| p_{R(\pi)}^{j}\left(\left|G_{\pi}\right|\right)\right)^{R(\pi)}} \leq p_{R(\pi)}^{j+1}\left(\left|G_{\pi}\right|\right) .
$$

The thesis follows by observing that $j \leq I(\pi)$.

This implies that every function which can be represented inside $\mathrm{RH}(A)$ is elementary time computable. 


\section{3 $\mathrm{RH}(\mathrm{W})$ and Polynomial Time}

Notice that the exponential bound of Proposition 4 has become a polynomial bound in Proposition 5 Since Proposition 4 has been the essential ingredient in proving the elementary bounds of Section 5.2 polynomial bounds are to be expected for $\mathrm{RH}(\mathrm{W})$. Indeed:

Theorem 4 For every $d, e \in \mathbb{N}$, there are polynomials $p_{e}^{d}: \mathbb{N} \rightarrow \mathbb{N}$ such that for every type derivation $\pi: \Gamma \vdash_{\mathrm{RH}(\mathrm{W})} M: A$, if $T \in T\left(G_{\pi}\right)$ then $|L(T)| \leq p_{R(\pi)}^{I(\pi)}(|M|)$.

Proof. We can proceed very similarly to the proof of Theorem 3 Consider the following polynomials:

$$
\begin{aligned}
\forall n, m \in \mathbb{N} \cdot p_{m}^{n} & : \mathbb{N} \rightarrow \mathbb{N} \\
p_{m}^{0}(x) & =x^{2} \\
p_{m}^{n+1}(x) & =x\left(x \cdot p_{m}^{n}(x)\right)^{n}
\end{aligned}
$$

For every $x, m, n, p_{m}^{n+1}(x) \geq p_{m}^{n}(x)$. We will prove that if $T=((t, e, U, L), f) \in T\left(G_{\pi}\right)$ is generated by $\mathbb{A}^{i}$, then $|t| \leq p_{R(\pi)}^{j}\left(\left|G_{\pi}\right|\right)$, where $j=\max \{I(\pi)-i, 0\}$. We go by induction on $j$.

If $j=0$, then $I(\pi) \leq i$. This implies that $|U(T)| \leq\left|G_{\pi}\right|$ by lemmas 1 and 6 similarly as in Theorem 3 As a consequence, $|t| \leq\left|G_{\pi}\right|^{2}=p_{R(\pi)}^{0}\left(\left|G_{\pi}\right|\right)$.

Now, suppose the thesis holds for $j$ and suppose $T=((t, e, U, L), f)$ to be guided by $\mathbb{A}^{i}$, where $R(\pi)-i=j+1$. By Lemma 6 and the induction hypothesis, $|U(T)| \leq\left(\left|G_{\pi}\right| p_{R(\pi)}^{j}\left(\left|G_{\pi}\right|\right)\right)^{R(\pi)}$, similarly as in theorem 3 As a consequence,

$$
\left.|t| \leq\left|G_{\pi}\right|\left(\left|G_{\pi}\right| p_{R(\pi)}^{j}\left(\left|G_{\pi}\right|\right)\right)^{R(\pi)} \leq p_{R(\pi)}^{j+1}\left(\left|G_{\pi}\right|\right)\right)
$$

The thesis follows by observing that $j \leq I(\pi)$.

\section{Embedding Complexity Classes}

In this section, we will provide embeddings of $\mathbf{F R}$ into $\mathrm{H}(\emptyset), \mathbf{F E}$ into $\mathrm{RH}(\mathrm{A})$ and $\mathbf{F P}$ into $\mathrm{RH}(\emptyset)$. This will complete the picture sketched in Section 3 First of all, we can prove that a weaker notion of contraction can be retrieved even if $\mathbf{D}=\emptyset$ :

Lemma 7 For every term $M$, there is a term $[M]_{x, y}^{w}$ such that for every $t \in \mathscr{E}_{\mathbb{U}},\left([M]_{x, y}^{w}\right)\{t / w\} \sim^{*} M\{t / x, t / y\}$. For every $n \in \mathbb{N}$, if $\Gamma, x: \mathbb{U}^{n}, y: \mathbb{U}^{n} \vdash_{\mathbf{H}(\emptyset)} M: A$ then $\Gamma, w: \mathbb{U}^{n} \vdash_{\mathbf{H}(\emptyset)}[M]_{x, y}^{w}: A$ and if $\Gamma, x: \mathbb{U}^{n}, y: \mathbb{U}^{n} \vdash_{\mathrm{RH}(\emptyset)} M: A$ then $\Gamma, w: \mathbb{U}^{n+1} \vdash_{\mathrm{RH}(\emptyset)}[M]_{x, y}^{w}: A$.

Proof. Given a term $t \in \mathscr{E} \mathbb{U}$, the term $\bar{t} \in \mathscr{E} \mathbb{C}$ is defined as follows, by induction on $t$ :

$$
\begin{aligned}
\overline{c_{2}^{\mathbb{U}}} & =c_{2}^{\mathbb{C}} ; \\
\overline{c_{1}^{\mathbb{U}} t} & =c_{1}^{\mathbb{C}} \bar{t} c_{2}^{\mathbb{C}} .
\end{aligned}
$$

We can define two closed terms Duplicate, Extract $\in \mathscr{M}_{\mathscr{A}}$ such that, for every $t \in \mathscr{E}_{\mathbb{U}}$

$$
\begin{aligned}
\text { Extract } \bar{t} & \sim^{*} t ; \\
\text { Duplicate } t & \sim^{*} \quad c_{2}^{\mathbb{C}} \bar{t} \bar{t} .
\end{aligned}
$$

The terms we are looking for are the following:

$$
\begin{aligned}
\text { Extract } & \equiv \lambda x \cdot x\left\langle\left\langle\lambda y \cdot \lambda w \cdot \lambda z \cdot \lambda q \cdot c_{1}^{\mathbb{U}} z, c_{2}^{\mathbb{U}}\right\rangle\right\rangle ; \\
\text { Duplicate } & \equiv \lambda x \cdot x\left\langle\left\langle\lambda y \cdot \lambda w \cdot w\left\{\left\{\lambda z \cdot \lambda q \cdot c_{1}^{\mathbb{C}}\left(c_{1}^{\mathbb{C}} z c_{2}^{\mathbb{C}}\right)\left(c_{1}^{\mathbb{C}} q c_{2}^{\mathbb{C}}\right), c_{2}^{\mathbb{C}}\right\}\right\}, c_{1}^{\mathbb{C}} c_{2}^{\mathbb{C}} c_{2}^{\mathbb{C}}\right\rangle\right\rangle .
\end{aligned}
$$


Indeed:

$$
\begin{aligned}
& c_{2}^{\mathbb{C}}\left\langle\left\langle\lambda y \cdot \lambda w \cdot \lambda z \cdot \lambda q \cdot c_{1}^{\mathbb{U}} z, c_{2}^{\mathbb{U}}\right\rangle\right\rangle \leadsto c_{2}^{\mathbb{U}} ; \\
& c_{1}^{\mathbb{C}} \bar{t} c_{2}^{\mathbb{C}}\left\langle\left\langle\lambda y \cdot \lambda w \cdot \lambda z \cdot \lambda q \cdot c_{1}^{\mathbb{U}} z, c_{2}^{\mathbb{U}\rangle\rangle} \sim^{*} \quad\left(\lambda y \cdot \lambda w \cdot \lambda z \cdot \lambda q \cdot c_{1}^{\mathbb{U}} z\right) \bar{t} c_{2}^{\mathbb{C}}\right.\right. \\
& \left(\bar{t}\left\langle\left\langle\lambda y \cdot \lambda w \cdot \lambda z \cdot \lambda q \cdot c_{1}^{\mathbb{U}} z, c_{2}^{\mathbb{U}}\right\rangle\right) c_{2}^{\mathbb{U}}\right. \\
& \sim^{*} \quad\left(\lambda y \cdot \lambda w \cdot \lambda z \cdot \lambda q \cdot c_{1}^{\mathbb{U}} z\right) \bar{t} c_{2}^{\mathbb{C}} t c_{2}^{\mathbb{U}} \\
& \sim^{*} \quad c_{1}^{\mathbb{U}} t \\
& \operatorname{Extract} \bar{t} \leadsto \bar{t}\left\langle\left\langle\lambda y \cdot \lambda w \cdot \lambda z \cdot \lambda q \cdot c_{1}^{\mathbb{U}} z, c_{2}^{\mathbb{U}}\right\rangle\right\rangle \\
& \sim^{*} t \\
& c_{2}^{\mathbb{U}}\left\langle\left\langle\lambda y \cdot \lambda w \cdot w\left\{\left\{\lambda z \cdot \lambda q \cdot c_{1}^{\mathbb{C}}\left(c_{1}^{\mathbb{C}} z c_{2}^{\mathbb{C}}\right)\left(c_{1}^{\mathbb{C}} q c_{2}^{\mathbb{C}}\right), c_{2}^{\mathbb{C}}\right\}\right\}, c_{1}^{\mathbb{C}} c_{2}^{\mathbb{C}} c_{2}^{\mathbb{C}}\right\rangle\right\rangle \sim^{*} \quad c_{1}^{\mathbb{C}} c_{2}^{\mathbb{C}} c_{2}^{\mathbb{C}} ; \\
& c_{1}^{\mathbb{U}} t\left\langle\left\langle\lambda y \cdot \lambda w \cdot w\left\{\left\{\lambda z \cdot \lambda q \cdot c_{1}^{\mathbb{C}}\left(c_{1}^{\mathbb{C}} z c_{2}^{\mathbb{C}}\right)\left(c_{1}^{\mathbb{C}} q c_{2}^{\mathbb{C}}\right), c_{2}^{\mathbb{C}}\right\}\right\}, c_{1}^{\mathbb{C}} c_{2}^{\mathbb{C}} c_{2}^{\mathbb{C}}\right\rangle\right\rangle \sim^{*} \quad c_{1}^{\mathbb{C}} \bar{t} \bar{t}\left\{\left\{\lambda z \cdot \lambda q \cdot c_{1}^{\mathbb{C}}\left(c_{1}^{\mathbb{C}} z c_{2}^{\mathbb{C}}\right)\left(c_{1}^{\mathbb{C}} q c_{2}^{\mathbb{C}}\right), c_{2}^{\mathbb{C}}\right\}\right\} \\
& \sim^{*} \quad c_{1}^{\mathbb{C}}\left(c_{1}^{\mathbb{C}} \bar{t} c_{2}^{\mathbb{C}}\right)\left(c_{1}^{\mathbb{C}} \bar{t} c_{2}^{\mathbb{C}}\right) \text {; } \\
& \text { Duplicate } t \leadsto t\left\langle\left\langle\lambda y \cdot \lambda w \cdot w \left\{\left\{\lambda z \cdot \lambda q \cdot c_{1}^{\mathbb{C}}\left(c_{1}^{\mathbb{C}} z c_{2}^{\mathbb{C}}\right)\left(c_{1}^{\mathbb{C}} q c_{2}^{\mathbb{C}}\right)\right.\right.\right.\right. \text {, } \\
& \left.\left.\left.\left.c_{2}^{\mathbb{C}}\right\}\right\}, c_{1}^{\mathbb{C}} c_{2}^{\mathbb{C}} c_{2}^{\mathbb{C}}\right\rangle\right\rangle \\
& \sim c_{1}^{\mathbb{C}} \bar{t} \bar{t} \text {. }
\end{aligned}
$$

Observe that, for every natural number $n$ :

$$
\begin{array}{rll}
\vdash_{\mathbf{H}(\emptyset)}: \text { Extract } & : & \mathbb{C}^{n} \multimap \mathbb{U}^{n} ; \\
\vdash_{\mathbf{H}(\emptyset)}: \text { Duplicate } & : & \mathbb{U}^{n} \multimap \mathbb{C}^{n} ; \\
\vdash_{\mathbf{H}(\emptyset)}: \text { Extract } & : & \mathbb{C}^{n+1} \multimap \mathbb{U}^{n} ; \\
\vdash_{\mathbf{H}(\emptyset)}: \text { Duplicate } & : & \mathbb{U}^{n+1} \multimap \mathbb{C}^{n} .
\end{array}
$$

Now let us define:

$$
[M]_{x, y}^{w} \equiv(\text { Duplicate } w)\{\{\lambda z \cdot \lambda q \cdot(\lambda x \cdot \lambda y \cdot M)(\text { Extract } z)(\text { Extract } q), \lambda x \cdot \lambda y \cdot M\}\}
$$

Indeed, for every $t \in \mathscr{E} \mathbb{U}$ :

$$
\begin{aligned}
& {[M]_{x, y}^{w}\{t / w\} \equiv(\text { Duplicate } t)\{\{\lambda z \cdot \lambda q \cdot(\lambda x \cdot \lambda y \cdot M)(\text { Extract } z)(\text { Extract } q), \lambda x . \lambda y \cdot M\}\}} \\
& \sim^{*}\left(c_{1}^{\mathbb{C}} \overline{t t}\right)\{\{\lambda z \cdot \lambda q \cdot(\lambda x \cdot \lambda y \cdot M)(\text { Extract } z)(\text { Extract } q), \lambda x \cdot \lambda y \cdot M\}\} \\
& \sim^{*}(\lambda x \cdot \lambda y \cdot M)(\text { Extract } \bar{t})(\text { Extract } \bar{t}) \\
& \sim^{*}(\lambda x \cdot \lambda y \cdot M) t t \\
& \sim \text { น } M\{t / x, t / y\} \text {. }
\end{aligned}
$$

Observe that the requirement of typings for $[M]_{x, y}^{w}$ can be easily verified.

The above lemma suffices to prove every primitive recursive function to be representable inside $H(\emptyset)$ :

Theorem 5 For every primitive recursive function $f: \mathbb{N}^{n} \rightarrow \mathbb{N}$ there is a term $M_{f}$ such that $\vdash_{\mathbf{H}(\emptyset)} M_{f}: \mathbb{U}^{0} \stackrel{n}{\circ} \mathbb{U}^{0}$ and $M_{f}$ represents $f$.

Proof. Base functions are the constant $0: \mathbb{N} \rightarrow \mathbb{N}$, the successor $s: \mathbb{N} \rightarrow \mathbb{N}$ and for every $n, i$ projections $u_{i}^{n}: \mathbb{N}^{n} \rightarrow \mathbb{N}$. It can be easily checked that these functions are represented by

$$
\begin{aligned}
M_{0} & \equiv \lambda x \cdot c_{2}^{\mathbb{U}} ; \\
M_{s} & \equiv \lambda x \cdot c_{1}^{\mathbb{U}} x ; \\
M_{u_{i}^{n}} & \equiv \lambda x_{1} \cdot \lambda x_{2} \ldots . \lambda x_{n} \cdot x_{i} .
\end{aligned}
$$




\section{Observe that}

$$
\begin{array}{rll}
\vdash_{\mathbf{H}(\emptyset)} M_{0} & : & \mathbb{U}^{0} \multimap \mathbb{U}^{0} ; \\
\vdash_{\mathbf{H}(\emptyset)} M_{s} & : & \mathbb{U}^{0} \multimap \mathbb{U}^{0} ; \\
\vdash_{\mathbf{H}(\emptyset)} M_{u_{i}^{n}} & : & \mathbb{U}^{0} \stackrel{n}{\circ} \mathbb{U}^{0} .
\end{array}
$$

We now need some additional notation. Given a term $M$ and $n$ variables $x_{1}, \ldots, x_{n}$, we will define terms $M_{i}^{x_{1}, \ldots, x_{n}}$ as follows:

$$
\begin{aligned}
M_{1}^{x_{1}, \ldots, x_{n}} & \equiv\left(\lambda x_{1} \ldots . \lambda x_{n} \cdot M\right) x_{1} \\
\forall i \geq 1 . M_{i+1}^{x_{1}, \ldots, x_{n}} & \equiv\left[M_{i}^{x_{1}, \ldots, x_{n}} x_{i+1}\right]_{x_{i}, x_{i+1}}^{x_{i+1}}
\end{aligned}
$$

We can prove the following by induction on $i$ :

$$
M_{i}^{x_{1}, \ldots, x_{n}}\left\{t / x_{i}\right\} \sim^{*}\left(\lambda x_{1} \ldots \lambda x_{n} . M\right) \underbrace{t \ldots t}_{i \text { times }} .
$$

Indeed:

$$
\begin{aligned}
M_{1}^{x_{1}, \ldots, x_{n}}\left\{t / x_{i}\right\} & \equiv\left(\lambda x_{1} \ldots . \lambda x_{n} . M\right) t \\
\forall i \geq 2 . M_{i+1}^{x_{1}, \ldots, x_{n}}\left\{t / x_{i+1}\right\} & \equiv\left[M_{i}^{x_{1}, \ldots, x_{n}} x_{i+1}\right]_{x_{i}, x_{i+1}}^{x_{i+1}}\left\{t / x_{i+1}\right\} \\
& \overbrace{}^{*}\left(M_{i}^{x_{1}, \ldots, x_{n}} x_{i+1}\right)\left\{t / x_{i}, t / x_{i+1}\right\} \\
& \overbrace{}^{*}\left(M_{i}^{x_{1}, \ldots, x_{n}}\left\{t / x_{i}\right\}\right) t \\
& \overbrace{}^{*}(\left(\lambda x_{1} \ldots \ldots \lambda x_{n} \cdot M\right) \underbrace{t \ldots t}_{i \text { times }}) t \\
& \equiv\left(\lambda x_{1} \ldots \lambda x_{n} . M\right) \underbrace{t \ldots t}_{i+1 \text { times }}
\end{aligned}
$$

In this way we can get a generalized variant of Lemma 7 by putting $\langle M\rangle_{x_{1}, \ldots, x_{n}}^{z} \equiv\left(\lambda x_{n} . M_{n}^{x_{1}, \ldots, x_{n}}\right) z$. Indeed:

$$
\begin{aligned}
\langle M\rangle_{x_{1}, \ldots, x_{n}}^{z}\{t / z\} & \equiv\left(\lambda x_{n} \cdot M_{n}^{x_{1}, \ldots, x_{n}}\right) t \\
& \leadsto M_{n}^{x_{1}, \ldots, x_{n}}\left\{t / x_{n}\right\} \\
& { }^{*}\left(\lambda x_{1} \ldots \ldots \lambda x_{n} \cdot M\right) \underbrace{t \ldots t}_{n \text { times }} \\
& \sim^{*} M\left\{t / x_{1}, \ldots t / x_{n}\right\} .
\end{aligned}
$$

We are now ready to prove that composition and recursion can be represented in $\mathbf{H}(\emptyset)$. Suppose $f: \mathbb{N}^{n} \rightarrow \mathbb{N}, g_{1}, \ldots, g_{n}$ : $\mathbb{N}^{m} \rightarrow \mathbb{N}$ and let $h: \mathbb{N}^{m} \rightarrow \mathbb{N}$ be the function obtained by composing $f$ with $g_{1}, \ldots, g_{n}$, i.e.

$$
h\left(n_{1}, \ldots, n_{m}\right)=f\left(g_{1}\left(n_{1}, \ldots, n_{m}\right), \ldots, g_{n}\left(n_{1}, \ldots, n_{m}\right)\right) .
$$

We define

$$
\begin{aligned}
N & \equiv \lambda x_{1}^{m} \ldots \lambda x_{n}^{m} \ldots \ldots \lambda x_{1}^{1} \ldots . \lambda x_{n}^{1} \cdot M_{f}\left(M_{g_{1}} x_{1}^{1} \ldots x_{1}^{m}\right) \ldots\left(M_{g_{n}} x_{n}^{1} \ldots x_{n}^{m}\right) \\
M_{h}^{m} & \equiv\left\langle N x_{1}^{m} \ldots x_{n}^{m}\right\rangle_{x_{1}^{m}, \ldots, x_{n}^{m}}^{y_{m}} \\
\forall i<m . M_{h}^{i} & \equiv\left\langle\lambda y_{i+1} \cdot\left(M_{h}^{i+1} x_{1}^{i} \ldots x_{n}^{i}\right)\right\rangle_{x_{1}^{i}, \ldots, x_{n}^{i}}^{y_{i}} \\
M_{h} & \equiv \lambda y_{1} \cdot M_{h}^{1} .
\end{aligned}
$$


Indeed:

$$
\begin{aligned}
M_{h}\left\ulcorner n_{1}\right\urcorner \ldots\left\ulcorner n_{m}\right\urcorner & \sim^{*}\left(M_{h}^{1}\left\{\left\ulcorner n_{1}\right\urcorner / y_{1}\right\}\right)\left\ulcorner n_{2}\right\urcorner \ldots\left\ulcorner n_{m}\right\urcorner \\
& \sim^{*}\left(\lambda y_{2+1} \cdot M_{h}^{2}\left\ulcorner n_{1}\right\urcorner \ldots\left\ulcorner n_{1}\right\urcorner\right)\left\ulcorner n_{2}\right\urcorner \ldots\left\ulcorner n_{m}\right\urcorner \\
& \leadsto \quad\left(M_{h}^{2}\left\{\left\ulcorner n_{2}\right\urcorner / y_{2}\right\}\left\ulcorner n_{1}\right\urcorner \ldots\left\ulcorner n_{1}\right\urcorner\right)\left\ulcorner n_{3}\right\urcorner \ldots\left\ulcorner n_{m}\right\urcorner \\
& { }^{*} \\
& \sim^{*}\left(\ldots\left(\left(N\left\ulcorner n_{m}\right\urcorner \ldots\left\ulcorner n_{m}\right\urcorner\right)\left\ulcorner n_{m-1}\right\urcorner \ldots\left\ulcorner n_{m-1}\right\urcorner\right) \ldots\right)\left\ulcorner n_{1}\right\urcorner \ldots\left\ulcorner n_{1}\right\urcorner \\
& { }^{*} \\
& M_{f}\left(M_{g_{1}}\left\ulcorner n_{1}\right\urcorner \ldots\left\ulcorner n_{m}\right\urcorner\right) \ldots\left(M_{g_{n}}\left\ulcorner n_{1}\right\urcorner \ldots\left\ulcorner n_{m}\right\urcorner\right) \\
& \left\ulcorner f\left(g_{1}\left(n_{1}, \ldots, n_{m}\right), \ldots, g_{n}\left(n_{1}, \ldots, n_{m}\right)\right)\right\urcorner .
\end{aligned}
$$

Now, suppose $f: \mathbb{N}^{m} \rightarrow \mathbb{N}$ and $g: \mathbb{N}^{m+2} \rightarrow \mathbb{N}$ and let $h: \mathbb{N}^{m+1} \rightarrow \mathbb{N}$ be the function obtained by $f$ and $g$ by primitive recursion, i.e.

$$
\begin{aligned}
h\left(0, n_{1}, \ldots, n_{m}\right) & =f\left(n_{1}, \ldots, n_{m}\right) \\
h\left(n+1, n_{1}, \ldots, n_{m}\right) & =g\left(n, h\left(n, n_{1}, \ldots, n_{m}\right), n_{1}, \ldots, n_{m}\right) .
\end{aligned}
$$

We define

$$
\begin{aligned}
N & \equiv \lambda x_{m} \cdot \lambda y_{m} \ldots . \lambda x_{1} \cdot \lambda y_{1} \cdot \lambda y \cdot \lambda w \cdot M_{g} y\left(w x_{1} \ldots x_{m}\right) y_{1} \ldots y_{m} ; \\
M_{h}^{m} & \equiv\left[N x_{m} y_{m}\right]_{x_{m}, y_{m}}^{z_{n}} ; \\
\forall i<m \cdot M_{h}^{i} & \equiv\left[\lambda z_{i+1} \cdot M_{h}^{i+1} x_{i} y_{i}\right]_{x_{i}, y_{i}}^{z_{i}} ; \\
N_{h} & \equiv \lambda y \cdot \lambda w \cdot \lambda z_{1} \ldots . \lambda z_{m} \cdot M_{h}^{1} z_{2} \ldots z_{n} y w ; \\
M_{h} & \equiv \lambda x \cdot x\left\langle\left\langle N_{h}, M_{f}\right\rangle .\right.
\end{aligned}
$$

Notice that:

$$
\begin{aligned}
\ulcorner 0\urcorner\left\langle\left\langle N_{h}, M_{f}\right\rangle\right\rangle & \leadsto M_{f} \equiv V_{0} ; \\
\ulcorner n+1\urcorner\left\langle\left\langle N_{h}, M_{f}\right\rangle\right\rangle & \leadsto N_{h}\ulcorner n\urcorner\left(\ulcorner n\urcorner\left\langle\left\langle N_{h}, M_{f}\right\rangle\right)\right) \\
& \leadsto * * \lambda z_{1} \ldots \lambda z_{m} \cdot M_{h}^{1} z_{2} \ldots z_{n}\ulcorner n\urcorner\left(\ulcorner n\urcorner\left\langle\left\langle N_{h}, M_{f}\right\rangle\right\rangle\right) \equiv V_{n} .
\end{aligned}
$$

and moreover, for every $\left\ulcorner n_{1}\right\urcorner, \ldots,\left\ulcorner n_{m}\right\urcorner$ :

$$
\begin{aligned}
& V_{0}\left\ulcorner n_{1}\right\urcorner \ldots\left\ulcorner n_{m}\right\urcorner \leadsto * \quad M_{f}\left\ulcorner n_{1}\right\urcorner \ldots\left\ulcorner n_{m}\right\urcorner \leadsto\left\ulcorner h\left(0, n_{1}, \ldots, n_{m}\right)\right\urcorner ; \\
& V_{n+1}\left\ulcorner n_{1}\right\urcorner \ldots\left\ulcorner n_{m}\right\urcorner \equiv\left(\lambda z_{1} \ldots \lambda z_{m} . M_{h}^{1} z_{2} \ldots z_{n}\ulcorner n\urcorner\left(\ulcorner n\urcorner\left\langle\left\langle N_{h}, M_{f}\right\rangle\right)\right)\right)\left\ulcorner n_{1}\right\urcorner \ldots\left\ulcorner n_{m}\right\urcorner \\
& \sim^{*} \quad M_{h}^{1}\left\{\left\ulcorner n_{1}\right\urcorner, z_{1}\right\}\left\ulcorner n_{2}\right\urcorner \ldots\left\ulcorner n_{m}\right\urcorner\ulcorner n\urcorner\left(\ulcorner n\urcorner\left\langle\left\langle N_{h}, M_{f}\right\rangle\right\rangle\right) \\
& \sim^{*} \quad\left(\lambda z_{2} \cdot M_{h}^{2}\left\ulcorner n_{1}\right\urcorner\left\ulcorner n_{1}\right\urcorner\right)\left\ulcorner n_{2}\right\urcorner \ldots\left\ulcorner n_{m}\right\urcorner\ulcorner n\urcorner\left(\ulcorner n\urcorner\left\langle\left\langle N_{h}, M_{f}\right\rangle\right\rangle\right) \\
& \sim^{*} \quad\left(\lambda z_{3} \cdot M_{h}^{3}\left\ulcorner n_{2}\right\urcorner\left\ulcorner n_{2}\right\urcorner\left\ulcorner n_{1}\right\urcorner\left\ulcorner n_{1}\right\urcorner\right)\left\ulcorner n_{3}\right\urcorner \ldots\ulcorner n\urcorner\left(\ulcorner n\urcorner\left\langle\left\langle N_{h}, M_{f}\right\rangle\right)\right. \\
& \sim^{*} \quad \ldots \\
& \sim^{*} \quad N\left\ulcorner n_{m}\right\urcorner\left\ulcorner n_{m}\right\urcorner \ldots\left\ulcorner n_{1}\right\urcorner\left\ulcorner n_{1}\right\urcorner\ulcorner n\urcorner\left(\ulcorner n\urcorner\left\langle\left\langle N_{h}, M_{f}\right\rangle\right\rangle\right) \\
& \sim^{*} \quad M_{g}\ulcorner n\urcorner\left(\ulcorner n\urcorner\left\langle\left\langle N_{h}, M_{f}\right\rangle\right\rangle\left\ulcorner n_{1}\right\urcorner \ldots\left\ulcorner n_{m}\right\urcorner\right)\left\ulcorner n_{1}\right\urcorner \ldots\left\ulcorner n_{m}\right\urcorner \\
& \text { 〜* } M_{g}\ulcorner n\urcorner\left(V_{n}\left\ulcorner n_{1}\right\urcorner \ldots\left\ulcorner n_{m}\right\urcorner\right)\left\ulcorner n_{1}\right\urcorner \ldots\left\ulcorner n_{m}\right\urcorner \\
& \sim^{*} \quad M_{g}\ulcorner n\urcorner\left\ulcorner h\left(n, n_{1}, \ldots, n_{m}\right)\right\urcorner\left\ulcorner n_{1}\right\urcorner \ldots\left\ulcorner n_{m}\right\urcorner \\
& \sim^{*}\left\ulcorner g\left(n, h\left(n, n_{1}, \ldots, n_{m}\right), n_{1}, \ldots, n_{m}\right)\right\urcorner \equiv\left\ulcorner h\left(n+1, n_{1}, \ldots, n_{m}\right)\right\urcorner .
\end{aligned}
$$

This concludes the proof.

The following two results show that functions representable in $\mathrm{RH}(\emptyset)$ (respectively, $\mathrm{RH}(\mathrm{A})$ ) combinatorially saturate $\mathbf{F P}$ (respectively, FE). 
Lemma 8 There are terms Coerc, Add, Square such that for every $n, m$

Coerc $\ulcorner n\urcorner \leadsto *\ulcorner n\urcorner$;

Add $\ulcorner n\urcorner\ulcorner m\urcorner \leadsto *\ulcorner n+m\urcorner$;

Square $\ulcorner n\urcorner \sim^{*}\left\ulcorner n^{2}\right\urcorner$.

Moreover, for every $i \in \mathbb{N}$

$$
\begin{array}{rll}
\vdash_{\mathrm{RH}(\emptyset)} \text { Coerc } & : & \mathbb{U}^{i+1} \multimap \mathbb{U}^{i} ; \\
\vdash_{\mathrm{RH}(\emptyset)} \text { Add } & : & \mathbb{U}^{i+1} \multimap \mathbb{U}^{i} \multimap \mathbb{U}^{i} ; \\
\vdash_{\mathrm{RH}(\emptyset)} \text { Square } & : & \mathbb{U}^{i+2} \multimap \mathbb{U}^{i} .
\end{array}
$$

Proof. Coerc is $\lambda x . x\left\langle\left\langle\lambda y \cdot \lambda w \cdot c_{1}^{\mathbb{U}} w, c_{2}^{\mathbb{U}}\right\rangle\right\rangle$. Add is $\lambda x \cdot \lambda y \cdot\left(x\left\langle\left\langle M_{1}, M_{2}\right\rangle\right\rangle\right) y$, where $M_{1}$ is $\lambda w \cdot \lambda z \cdot \lambda q \cdot c_{1}^{\mathbb{U}}(z q)$ and $M_{2}$ is $\lambda z . z$. Square is

$$
\lambda x \cdot\left[\operatorname{Add}\left(\left(\operatorname{Coerc} x_{1}\right)\left\langle\left\langle\text { Add }, c_{2}^{\mathbb{U}},\right\rangle\right\rangle\right)\left(\left(\text { Predecessor } x_{2}\right)\left\langle\left\langle\operatorname{Add}, c_{2}^{\mathbb{U}}\right\rangle\right\rangle\right)\right]_{x_{1}, x_{2}}^{x}
$$

where Predecessor is $\lambda x . x\left\{\left\{\lambda y . y, c_{2}^{\mathbb{U}}\right\}\right\}$. Indeed:

$$
\begin{aligned}
& c_{2}^{\mathbb{U}}\left\langle\left\langle\lambda y \cdot c_{1}^{\mathbb{U}} y, c_{2}^{\mathbb{U}}\right\rangle\right\rangle \leadsto c_{2}^{\mathbb{U}} ; \\
& c_{1}^{\mathbb{U}} t\left\langle\left\langle\lambda y \cdot \lambda w \cdot c_{1}^{\mathbb{U}} w, c_{2}^{\mathbb{U}}\right\rangle\right\rangle \leadsto\left(\lambda y \cdot \lambda w \cdot c_{1}^{\mathbb{U}} w\right) t\left(t\left\langle\left\langle\lambda y \cdot \lambda w \cdot c_{1}^{\mathbb{U}} w, c_{2}^{\mathbb{U}}\right\rangle\right\rangle\right) \\
& \sim^{*}\left(\lambda y \cdot \lambda w \cdot c_{1}^{\mathbb{U}} w\right) t t \\
& \sim^{*} \quad c_{1}^{\mathbb{U}} t \\
& \operatorname{Coerc} t \leadsto t\left\langle\left\langle\lambda y \cdot \lambda w \cdot c_{1}^{\mathbb{U}} w, c_{2}^{\mathbb{U}}\right\rangle\right\rangle \sim^{*} t ; \\
& \ulcorner 0\urcorner\left\langle\left\langle M_{1}, M_{2}\right\rangle\right\rangle \leadsto \lambda y \cdot y \equiv V_{0} ; \\
& \ulcorner n+1\urcorner\left\langle\left\langle M_{1}, M_{2}\right\rangle\right\rangle \quad \leadsto \quad M_{1}\ulcorner n\urcorner\left(\ulcorner n\urcorner\left\langle\left\langle M_{1}, M_{2}\right\rangle\right\rangle\right) \\
& \leadsto \quad\left(\lambda z \cdot \lambda q \cdot c_{1}^{\mathbb{U}}(z q)\right) V_{n} \leadsto \lambda q \cdot c_{1}^{\mathbb{U}}\left(V_{n} q\right) \equiv V_{n+1} ; \\
& V_{0}\ulcorner m\urcorner \leadsto\ulcorner m\urcorner \text {; } \\
& V_{n+1}\ulcorner m\urcorner \quad \leadsto \quad c_{1}^{\mathbb{U}}\left(V_{n}\ulcorner m\urcorner\right) \sim^{*} c_{1}^{\mathbb{U}}\ulcorner n+m\urcorner \equiv\ulcorner(n+1)+m\urcorner ; \\
& \operatorname{Add}\ulcorner n\urcorner\ulcorner m\urcorner \leadsto\left(\ulcorner n\urcorner\left\langle\left\langle M_{1}, M_{2}\right\rangle\right\rangle\right)\ulcorner m\urcorner \\
& \sim^{*} \quad V_{n}\ulcorner m\urcorner \sim^{*}\ulcorner n+m\urcorner \text {; } \\
& \ulcorner 0\urcorner\left\langle\left\langle\mathbf{A d d}, c_{2}^{\mathbb{U}}\right\rangle\right\rangle \leadsto\ulcorner 0\urcorner \equiv\ulcorner 0(0+1) / 2\urcorner ; \\
& \ulcorner n+1\urcorner\left\langle\left\langle\mathbf{A d d}, c_{2}^{\mathbb{U}}\right\rangle\right\rangle \leadsto \mathbf{A d d}\ulcorner n\urcorner\left(\ulcorner n\urcorner\left\langle\left\langle\mathbf{A d d}, c_{2}^{\mathbb{U}}\right\rangle\right)\right. \\
& \leadsto \mathbf{A d d}\ulcorner n\urcorner(\ulcorner n(n+1) / 2\urcorner) \sim^{*}\ulcorner n+n(n+1) / 2\urcorner \equiv\ulcorner(n+1)(n+2) / 2\urcorner ; \\
& \text { Square }\ulcorner 0\urcorner \sim^{*} \operatorname{Add}\left((\operatorname{Coerc}\ulcorner 0\urcorner)\left\langle\left\langle\operatorname{Add}, c_{2}^{\mathbb{U}},\right\rangle\right\rangle\right)\left((\text { Predecessor }\ulcorner 0\urcorner)\left\langle\left\langle\operatorname{Add}, c_{2}^{\mathbb{U}}\right\rangle\right\rangle\right) \\
& \sim^{*} \quad \operatorname{Add}\left(\ulcorner 0\urcorner\left\langle\left\langle\mathbf{A d d}, c_{2}^{\mathbb{U}},\right\rangle\right\rangle\right)\left(\ulcorner 0\urcorner\left\langle\left\langle\mathbf{A d d}, c_{2}^{\mathbb{U}}\right\rangle\right\rangle\right) \sim^{*} \operatorname{Add}\ulcorner 0\urcorner\ulcorner 0\urcorner \\
& \sim^{*}\ulcorner 0\urcorner \text {; } \\
& \text { Square }\ulcorner n+1\urcorner \sim^{*} \operatorname{Add}\left((\operatorname{Coerc}\ulcorner n+1\urcorner)\left\langle\left\langle\operatorname{Add}, c_{2}^{\mathbb{U}},\right\rangle\right\rangle\right) \\
& \left((\text { Predecessor }\ulcorner n+1\urcorner)\left\langle\left\langle\text { Add }, c_{2}^{\mathbb{U}}\right\rangle\right\rangle\right) \\
& \sim^{*} \quad \operatorname{Add}\left(\ulcorner n+1\urcorner\left\langle\left\langle\mathbf{A d d}, c_{2}^{\mathbb{U}},\right\rangle\right\rangle\right)\left(\ulcorner n\urcorner\left\langle\left\langle\mathbf{A d d}, c_{2}^{\mathbb{U}}\right\rangle\right\rangle\right) \\
& \sim^{*} \quad \boldsymbol{A d d}\ulcorner(n+1)(n+2) / 2\urcorner\ulcorner n(n+1) / 2\urcorner \\
& \sim^{*}\ulcorner(n+1)(n+2) / 2+n(n+1) / 2\urcorner \equiv\left\ulcorner n^{2}\right\urcorner \text {. }
\end{aligned}
$$

This concludes the proof.

In presence of ramification, an exponential behavior can be obtained by exploiting contraction on tree-algebraic types:

Lemma 9 There is a term $\operatorname{Exp}$ such that for every $n$

$$
\operatorname{Exp}\ulcorner n\urcorner \sim^{*}\left\ulcorner 2^{n}\right\urcorner .
$$

Moreover, for every $i \in \mathbb{N}$

$$
\vdash_{\mathrm{RH}(\mathrm{A})} \operatorname{Exp}: \mathbb{U}^{i+2} \multimap \mathbb{U}^{i}
$$


Proof. For every $n \in \mathbb{N}$, we will denote by $\operatorname{ct}(n)$ the complete binary tree of height $n$ in $\mathscr{E}_{\mathbb{C}}$ :

$$
\begin{aligned}
c t(0) & =c_{2}^{\mathbb{C}} ; \\
c t(n+1) & =c_{1}^{\mathbb{C}}(c t(n))(c t(n)) .
\end{aligned}
$$

For every $n$, there are $2^{n}$ instances of $c_{2}^{\mathbb{C}}$ inside $c t(n)$. We will now define two terms Blowup and Leaves such that

$$
\begin{array}{rll}
\text { Blowup }\ulcorner n\urcorner & \sim^{*} & c t(n) ; \\
\text { Leaves }(c t(n)) & \sim^{*} \quad\left\ulcorner 2^{n}\right\urcorner .
\end{array}
$$

We define:

$$
\begin{aligned}
\text { Blowup } & \equiv \lambda x \cdot x\left\langle\left\langle\lambda y \cdot \lambda w \cdot c_{1}^{\mathbb{C}} w w, c_{2}^{\mathbb{C}}\right\rangle\right\rangle \\
\text { Leaves } & \equiv \lambda x \cdot\left(x\left\langle\left\langle\lambda y \cdot \lambda w \cdot \lambda z \cdot \lambda q \cdot \lambda r \cdot z(q r) \cdot \lambda x \cdot c_{1}^{\mathbb{U}} x\right\rangle\right)\right) c_{2}^{\mathbb{U}} \\
\text { Exp } & \equiv \lambda x \cdot \operatorname{Leaves}(\operatorname{Blowup} x) .
\end{aligned}
$$

Indeed:

$$
\begin{aligned}
& \ulcorner 0\urcorner\left\langle\left\langle\lambda y \cdot c_{1}^{\mathbb{C}} y y, c_{2}^{\mathbb{C}}\right\rangle\right\rangle \leadsto c_{1}^{\mathbb{C}} \equiv \operatorname{ct}(0) ; \\
& \ulcorner n+1\urcorner\left\langle\left\langle\lambda y \cdot \lambda w \cdot c_{1}^{\mathbb{C}} w w, c_{2}^{\mathbb{C}}\right\rangle\right\rangle \leadsto\left(\lambda y \cdot \lambda w \cdot c_{1}^{\mathbb{C}} w w, c_{2}^{\mathbb{C}}\right)\ulcorner n\urcorner\left(\ulcorner n\urcorner\left\langle\left\langle\lambda y \cdot \lambda w \cdot c_{1}^{\mathbb{C}} w w, c_{2}^{\mathbb{C}}\right\rangle\right)\right. \\
& \sim^{*} \quad\left(\lambda y \cdot \lambda w \cdot c_{1}^{\mathbb{C}} w w, c_{2}^{\mathbb{C}}\right)\ulcorner n\urcorner(c t(n)) ; \\
& \sim^{*} \quad c_{1}^{\mathbb{C}}(\operatorname{ct}(n))(\operatorname{ct}(n)) \equiv \operatorname{ct}(n+1) \\
& \text { Blowup }\ulcorner n\urcorner \leadsto\ulcorner n\urcorner\left\langle\left\langle\lambda y \cdot c_{1}^{\mathbb{C}} y y, c_{2}^{\mathbb{C}}\right\rangle\right\rangle \sim(c t(n)) ; \\
& (c t(0))\left\langle\left\langle\lambda y \cdot \lambda w \cdot \lambda z \cdot \lambda q \cdot \lambda r \cdot z(q r) \cdot \lambda x \cdot c_{1}^{\mathbb{U}} x\right\rangle\right\rangle \quad \leadsto \quad \lambda x \cdot c_{1}^{\mathbb{U}} x \equiv V_{0} ; \\
& (c t(n+1))\left\langle\left\langle\lambda y \cdot \lambda w \cdot \lambda z \cdot \lambda q \cdot \lambda r \cdot z(q r) \cdot \lambda x \cdot c_{1}^{\mathbb{U}} x\right\rangle\right\rangle \quad \sim^{*} \quad \lambda r \cdot V_{n}\left(V_{n} r\right) \equiv V_{n+1} ; \\
& V_{0}\ulcorner m\urcorner \leadsto\ulcorner 1+m\urcorner \equiv\left\ulcorner 2^{0}+m\right\urcorner ; \\
& V_{n+1}\ulcorner m\urcorner \quad \leadsto \quad V_{n}\left(V_{n}\ulcorner m\urcorner\right) \sim^{*} V_{n}\left\ulcorner 2^{n}+m\right\urcorner \\
& \sim^{*}\left\ulcorner 2^{n}+2^{n}+m\right\urcorner \equiv\left\ulcorner 2^{n+1}+m\right\urcorner ; \\
& \text { Leaves }(c t(n)) \leadsto\left((c t(n))\left\langle\left\langle\lambda y \cdot \lambda w \cdot \lambda z \cdot \lambda q \cdot \lambda r \cdot z(q r) \cdot \lambda x \cdot c_{1}^{\mathbb{U}} x\right\rangle\right)\right) c_{2}^{\mathbb{U}} \\
& \sim^{*} \quad V_{n}\ulcorner 0\urcorner \sim *\left\ulcorner 2^{n}\right\urcorner ; \\
& \operatorname{Exp}\ulcorner n\urcorner \leadsto \lambda x \text {.Leaves(Blowup }\ulcorner n\urcorner) \sim^{*} \text { Leaves }(c t(n)) \\
& \sim^{*}\left\ulcorner 2^{n}\right\urcorner \text {. }
\end{aligned}
$$

This concludes the proof.

The last two lemmata are not completeness results, but help in the so-called quantitative part of the encoding of Turing Machines. Indeed, $\mathbf{F P}$ can be embedded into $\mathrm{RH}(\emptyset)$, while $\mathbf{F E}$ can be embedded into $\mathrm{RH}(\mathrm{A})$ :

Theorem 6 For every polynomial time computable function $f:\{0,1\}^{*} \rightarrow\{0,1\}^{*}$ there are a term $M_{f}$ and an integer $n_{f}$ such that $\vdash_{\mathrm{RH}(\emptyset)} M_{f}: \mathbb{B}^{n_{f}} \rightarrow \mathbb{B}^{0}$ and $M_{f}$ represents $f$. For every elementary time computable function $f:\{0,1\}^{*} \rightarrow$ $\{0,1\}^{*}$ there are a term $M_{f}$ and an integer $n_{f}$ such that $\vdash_{\mathrm{RH}(\mathrm{A})} M_{f}: \mathbb{B}^{n_{f}} \rightarrow \mathbb{B}^{0}$ and $M_{f}$ represents $f$.

Proof. First of all, we can observe that for ever polynomial $p: \mathbb{N} \rightarrow \mathbb{N}$, there are another polynomial $\bar{p}: \mathbb{N} \rightarrow \mathbb{N}$, an integer $n_{p}$ and term $M_{\bar{p}}$ such that

$$
\begin{aligned}
\forall n \in \mathbb{N} . \bar{p}(n) \quad \geq \quad p(n) ; \\
\forall n \in \mathbb{N} . \quad \vdash_{\mathrm{RH}(\emptyset)} \quad M_{\bar{p}}: \mathbb{B}^{n_{p}+n} \multimap \mathbb{B}^{n} ;
\end{aligned}
$$

and $M_{\bar{p}}$ represents $\bar{p} . \bar{p}$ is simply $p$ where all monomials $x^{k}$ are replaced by $x^{2^{l}}$ (where $k \leq 2^{l}$ ) and $M_{\bar{p}}$ is built up from terms in $\mathscr{E}_{\mathbb{U}}$, Add, Square and Coerc (see Lemma 8 ). Analogously, for every elementary function $p: \mathbb{N} \rightarrow \mathbb{N}$, there are another elementary function $\bar{p}: \mathbb{N} \rightarrow \mathbb{N}$, an integer $n_{p}$ and term $M_{\bar{p}}$ such that

$$
\begin{aligned}
& \forall n \in \mathbb{N} \cdot \bar{p}(n) \quad \geq \quad p(n) ; \\
& \forall n \in \mathbb{N} . \quad \vdash_{\mathrm{RH}(\mathrm{A})} \quad M_{\bar{p}}: \mathbb{B}^{n_{p}+n} \multimap \mathbb{B}^{n} .
\end{aligned}
$$


and $M_{\bar{p}}$ represents $\bar{p}$. This time, $\bar{p}$ is a tower

$$
\left.\bar{p}(n)=2 \cdot .^{2^{n}}\right\} k \text { times }
$$

obtained from $p$ by applying a classical result on elementary functions, while $M_{\bar{p}}$ built up from terms in $\mathscr{E} \mathbb{U}$, Add, Coerc and $\operatorname{Exp}$ (see Lemma 9 ).

Now, consider a Turing Machine $\mathcal{M}$ working in polynomial time. Configurations for $\mathcal{M}$ are quadruples (state, left, right, current), where state belongs to a finite set of states, left, right $\in \Sigma^{*}$ (where $\Sigma$ is a finite alphabet) are the contents of the left and right portion of the tape, and current $\in \Sigma$ is the symbol currently read by the head. It it not difficult to encode configurations of $\mathcal{M}$ by terms in $\mathscr{E} \mathbb{D}$ in such a way that terms $M_{\text {init }}, M_{\text {final }}, M_{\text {trans }}$ exists such that:

- $M_{\text {init }}\ulcorner s\urcorner$ rewrites to the term encoding the initial configuration on $s, M_{\text {final }}$ extract the result from a final configuration and $M_{\text {trans }}$ represents the transition function of $\mathcal{M}$;

- For every $n$,

$$
\begin{aligned}
& \vdash_{\mathrm{RH}(\emptyset)} M_{\text {init }} \quad: \quad \mathbb{B}^{n+1} \multimap \mathbb{D}^{n} ; \\
& \vdash_{\mathrm{RH}(\emptyset)} M_{\text {final }}: \mathbb{D}^{n+1} \multimap \mathbb{B}^{n} \text {; } \\
& \vdash_{\mathrm{RH}(\emptyset)} M_{\text {trans }}: \mathbb{D}^{n} \multimap \mathbb{D}^{n} \text {. }
\end{aligned}
$$

Moreover, there is a term $M_{\text {length }}$ such that $M_{\text {length }}\ulcorner s\urcorner \sim^{*}\ulcorner|s|\urcorner$ for every $s \in\{0,1\}^{*}$. Let now $p: \mathbb{N} \rightarrow \mathbb{N}$ be a polynomial bounding the running time of $\mathcal{M}$. The function computed by $\mathcal{M}$ is the one represented by the term:

$$
M_{\mathcal{M}} \equiv \lambda x .\left\langle M_{\text {final }}\left(\left(\left(M_{\bar{p}}\left(M_{\text {length }} y\right)\right)\left\langle\left\langle\lambda x . \lambda y \cdot \lambda w \cdot y\left(M_{\text {trans }} w\right), \lambda x . x\right\rangle\right\rangle\right)\left(M_{\text {init }} z\right)\right)\right\rangle_{y, z}^{x}
$$

where $\langle M\rangle_{y, z}^{x}$ is the generalization of $[M]_{x, y}^{z}$ to the algebra $\mathbb{B}$.

If $\mathcal{M}$ works in elementary time, we can proceed in the same way.

\section{Conclusions}

We introduced a typed lambda-calculus equivalent to Gödel System T and a new context-based semantics for it. We then characterized the expressive power of various subsystems of the calculus, all of them being obtained by imposing linearity and ramification constraints. To the author's knowledge, the only fragment whose expressive power has been previously characterized is $\mathrm{RH}(\mathrm{W})$ (see [17, 4, 8]). In studying the combinatorial dynamics of normalization, the semantics has been exploited in an innovative way.

There are other systems to which our semantics can be applied. This, in particular, includes non-size-increasing polynomial time computation [16] and the calculus capturing NC by Aehlig et al. [1]. Moreover, we believe higher-order contraction can be accomodated in the framework by techiques similar to the ones from [15].

The most interesting development, however, consists in studying the applicability of our semantics to the automatic extraction of runtime bounds from programs. This, however, goes beyond the scope of this paper and is left to future investigations.

\section{Acknowledgements}

The author would like to thank Simone Martini and Luca Roversi for their support and the anonymous referees for many useful comments.

\section{References}

[1] K. Aehlig, J. Johannsen, H. Schwichtenberg, and S. A. Terwijn. Linear ramified higher type recursion and parallel complexity. In Proof Theory in Computer Science, volume 2183 of LNCS, pages 1-21. 2001.

[2] P. Baillot and M. Pedicini. Elementary complexity and geometry of interaction. Fundamenta Informaticae, 45(1-2):1-31, 2001.

[3] S. Bellantoni and S. Cook. A new recursion-theoretic characterization of the polytime functions. Computational Complexity, 2:97110, 1992.

[4] S. Bellantoni, K. H. Niggl, and H. Schwichtenberg. Higher type recursion, ramification and polynomial time. Annals of Pure and Applied Logic, 104:17-30, 2000. 
[5] G. Bonfante, J.-Y. Marion, and J.-Y. Moyen. On complexity analysis by quasi-interpretations. Theoretical Computer Science, 2004. To appear.

[6] U. Dal Lago. Context semantics, linear logic and computational complexity. In Proc. 21th IEEE Syposium on Logic in Computer Science, pages 169-178, 2006.

[7] U. Dal Lago and S. Martini. An invariant cost model for the lambda calculus. In Proc. Second Conference on Computability in Europe, pages 105-114, 2006.

[8] U. Dal Lago, S. Martini, and L. Roversi. Higher order linear ramified recurrence. In Types for Proofs and Programs, Post-Workshop Proceedings, volume 3085 of LNCS, pages 178-193, 2003.

[9] V. Danos, H. Herbelin, and L. Regnier. Game semantics \& abstract machines. In Proc. 11th IEEE Syposium on Logic in Computer Science, pages 394-405, 1996.

[10] V. Danos and L. Regnier. Reversible, irreversible and optimal lambda-machines. Theoretical Computer Science, 227(1-2):79-97, 1999.

[11] D. Ghica. Slot games: A quantitative model of computation. In Proc. 32nd ACM Symposium on Principles of Programming Languages, pages 85-97, 2005.

[12] J.-Y. Girard. Geometry of interaction 2: deadlock-free algorithms. In Proc. Conference on Computer Logic, volume 417 of LNCS, pages 76-93, 1988.

[13] J.-Y. Girard. Geometry of interaction 1: interpretation of system F. In Proc. Logic Colloquium '88, pages 221-260, 1989.

[14] J.-Y. Girard. Light linear logic. Information and Computation, 143(2):175-204, 1998.

[15] G. Gonthier, M. Abadi, and J.-J. Lévy. The geometry of optimal lambda reduction. In Proc. 12th ACM Symposium on Principles of Programming Languages, pages 15-26, 1992.

[16] M. Hofmann. Linear types and non-size-increasing polynomial time computation. In Proc. 14th IEEE Symposium on Logic in Computer Science, pages 464-473, 1999.

[17] M. Hofmann. Safe recursion with higher types and BCK-algebra. Annals of Pure and Applied Logic, 104:113-166, 2000.

[18] F. Joachimski and R. Matthes. Short proofs of normalization for the simply-typed lambda-calculus, permutative conversions and Gödel's T. Archive for Mathematical Logic, 42(1):59-87, 2003.

[19] Y. Lafont. Soft linear logic and polynomial time. Theoretical Computer Science, 318:163-180, 2004.

[20] D. Leivant. Stratified functional programs and computational complexity. In Proc. 20th ACM Symposium on Principles of Programming Languages, pages 325-333, 1993.

[21] D. Leivant. Applicative control and computational complexity. In Proc. 13th International Workshop on Computer Science Logic, volume 1685 of $L N C S$, pages 82-95, 1999.

[22] D. Leivant. Ramified recurrence and computational complexity III: Higher type recurrence and elementary complexity. Annals of Pure and Applied Logic, 96:209-229, 1999.

[23] D. Leivant. Intrinsic reasoning about functional programs I: first order theories. Annals of Pure and Applied Logic, 114(1-3):117-153, 2002.

[24] D. Leivant. Intrinsic reasoning about functional programs II: unipolar induction and primitive-recursion. Theoretical Computer Science, 318(1-2):181-196, 2004.

[25] D. Leivant and J.-Y. Marion. Ramified recurrence and computational complexity II: Substitution and poly-space. In Proc. 8th International Workshop on Computer Science Logic, volume 933 of LNCS, pages 486-500, 1994.

[26] H. Mairson. From Hilbert spaces to Dilbert spaces: Context semantics made simple. In Proc. 22nd Conference on Foundations of Software Technology and Theoretical Computer Science, volume 2556 of LNCS, pages 2-17, 2002.

[27] G. Ostrin and S. Wainer. Proof theoretic complexity. In H. Schwichtenberg and R. Steinbrüggen, editors, Proof and System Reliability, volume 62 of NATO Science Series, pages 369-398. Kluwer, 2002.

[28] D. Sands. Operational theories of improvement in functional languages. In Proc. 1991 Glasgow Workshop on Functional Programming, pages 298-311, 1991.

[29] H. Simmons. Tiering as a recursion technique. Bulletin of Symbolic Logic, 11(3):321-350, 2005. 